\title{
Semiclassical basis sets for the computation of molecular vibrational states
}

\author{
F. Revuelta, ${ }^{1,2, a)}$ E. Vergini, $\left.{ }^{3, b}\right)$ R. M. Benito, ${ }^{1, c)}$ and F. Borondo ${ }^{2,4, d)}$ \\ ${ }^{1}$ Grupo de Sistemas Complejos, Escuela Técnica Superior de Ingeniería Agronómica, \\ Alimentaria y de Biosistemas, Universidad Politécnica de Madrid, 28040 Madrid, Spain \\ ${ }^{2}$ Instituto de Ciencias Matemáticas (ICMAT), 28049 Cantoblanco, Madrid, Spain \\ ${ }^{3}$ Departamento de Física, Comisión Nacional de Energía Atómica, Av. del Libertador 8250 , \\ 1429 Buenos Aires, Argentina \\ ${ }^{4}$ Departamento de Química, Universidad Autónoma de Madrid, 28049 Cantoblanco, Madrid, Spain
}

(Received 10 October 2016; accepted 14 December 2016; published online 5 January 2017)

\begin{abstract}
In this paper, we extend a method recently reported [F. Revuelta et al., Phys. Rev. E 87, 042921 (2013)] for the calculation of the eigenstates of classically highly chaotic systems to cases of mixed dynamics, i.e., those presenting regular and irregular motions at the same energy. The efficiency of the method, which is based on the use of a semiclassical basis set of localized wave functions, is demonstrated by applying it to the determination of the vibrational states of a realistic molecular system, namely, the LiCN molecule. Published by AIP Publishing. [http://dx.doi.org/10.1063/1.4973376]
\end{abstract}

\section{INTRODUCTION}

The quantum description of physical and chemical processes customarily pivots around the determination of the eigenenergies and eigenfunctions of the system. Except in the particular case of separable Hamiltonians, one has to resort to numerical computation for this important task, and numerous procedures have been designed for this end. ${ }^{1-3}$ Moreover, this problem is particularly demanding in the classical limit, i.e., $\hbar \rightarrow 0$, where the density of states is high, and also in realistic systems, which usually exhibit a classically chaotic dynamical behavior even for the modest values of the excitation energy. When this happens in time-reversal systems, most eigenfunctions present a very complex nodal pattern that can be adequately described only by using large basis sets, usually making computations extremely time consuming.

In this respect, semiclassical methods ${ }^{4}$ can be very helpful, both at the computational level and also providing valuable help in the understanding of the correspondence between classical and quantum mechanics. These methods are based on the classical underlying properties of the system and constitute a cornerstone in the study of classically chaotic systems. In the presence of chaos, the traditional Wentzel-Kramers-Brillouin (WKB) or Einstein-Brillouin-Keller (EBK) approximations cannot be applied due to the absence of the invariant tori ${ }^{4,5}$ that provide the support for the corresponding wave functions. Nevertheless, classical periodic orbits (POs) have a profound impact on the (quantum) density of states of the system, as shown by Gutzwiller in 1971 with his celebrated (semiclassical) trace formula. ${ }^{5}$ Unfortunately, the application of this expression to the calculation of highly excited states is very limited due to the exponential proliferation in the number of POs as energy increases.

\footnotetext{
${ }^{\text {a) E-mail: fabio.revuelta@upm.es }}$

b) E-mail: vergini@tandar.cnea.gov.ar

c) E-mail: rosamaria.benito@upm.es

d) E-mail: f.borondo@uam.es
}

The importance of unstable POs for some individual eigenfunctions of classically chaotic systems is clear after the seminal work of Heller on scarring. ${ }^{6}$ In that paper, the author coined the term scar to refer to an enhanced localization (over the statistically expected value ${ }^{7}$ ) of the quantum probability in some eigenfunctions along periodic trajectories. Actually, scars are associated with Bohr-Sommerfeld (BS) quantized short POs. However, this is a necessary but not a sufficient condition for their appearance which, as a consequence, cannot be predicted. Scars have been studied theoretically in quantum billiards, ${ }^{8}$ anharmonic molecular potentials, ${ }^{9-20}$ or quantum maps. ${ }^{21}$ Also, they have been observed in the laboratory in different microwave, ${ }^{22,23}$ optical fibers, ${ }^{24}$ microcavities, ${ }^{25}$ solid state devices, ${ }^{26}$ graphene, ${ }^{27}$ or ultracold atom experiments. ${ }^{28}$

Several important results on scarring have been reported in the literature. For example, Bogomolny demonstrated how scars are in general "distributed" among groups of individual eigenfunctions, and scarred functions can also be produced in the semiclassical limit by averaging of a number of neighbor eigenfunctions around the BS quantized energies $^{29}$ (see also Ref. 30). Later, Berry ${ }^{31}$ demonstrated by using Wigner functions that this localization does not only take place in the configuration space but also in the phase space. Prado and Keating ${ }^{32}$ showed that the scarring localization is enhanced in the presence of bifurcations in systems with mixed dynamics, giving rise to the so-called superscars. Going beyond the influence of POs in the quantum mechanics of chaotic systems, the effect of the recurrences over homoclinic and heteroclinic quantized circuits has also been reported in the literature. ${ }^{33}$ Finally, scarring in open systems has been studied. $^{34}$

Several methods have been proposed to construct localized wave functions over unstable POs, usually known as "scar functions." For example, de Polavieja et al. averaged groups of eigenfunctions by performing a short-time quantum evolution, ${ }^{30}$ and Vergini and co-workers ${ }^{35}$ combined PO resonances by minimizing energy dispersion, then including 
the semiclassical dynamics around the scarring PO up to the Ehrenfest time. ${ }^{36}$ More recently, Sibert et al. ${ }^{37}$ and Revuelta et al. ${ }^{19,38}$ applied these ideas to systems with smooth potentials, and Vagov et al. ${ }^{39}$ extended the asymptotic boundary layer method to calculate stable microresonator localized modes over unstable POs.

Scar functions have a very interesting and useful property, aside from their spatial localization: they also present a very low dispersion in energy. We have recently used this fact to construct an extremely efficient basis set for the diagonalization of the Hamiltonian matrix in a coupled quartic oscillator with a high degree of chaoticity. As demonstrated in Ref. 40, the system eigenfunctions can be obtained from a very small number of scar functions, i.e., POs, thus getting around somehow the exponential growth fate of the Gutzwiller theory. This is based on the replacement of the longer POs by the interaction of the shorter ones. This reduces dramatically the basis size, which in our method only increases linearly with the number of accurately calculated eigenfunctions.

The aim of this paper is to demonstrate the feasibility of extending the method reported in Ref. 40 to systems of chemical interest with a mixed phase space, where trajectories with regular and irregular motions coexist at the same energy. For this purpose, we show how to construct an efficient semiclassical basis set formed by localized wave functions, using the method originally reported in Refs. 19 and 38 which is used to compute the vibrational eigenstates of the $\mathrm{LiNC} / \mathrm{LiCN}$ isomerizing system. The method is performed in two steps: (1) construction of localized wave functions on POs ("tube" functions for the stable and "scar" functions for the unstable POs), and (2) application of a modified version of Gram-Schmidt orthogonalization to construct an efficient basis set, where the Hamiltonian matrix is computed and diagonalized. The method relies on some concepts of the chaos theory, such us stability analysis or bifurcation diagrams, that can be found, for example, in Ref. 41.

The paper is organized as follows. In Sec. II, we introduce the system under study. In Sec. III we describe the method that we have developed for the computation of the eigenenergies and eigenfunctions of a system presenting coexisting regions of regular and irregular motion, using a basis set of scar wave functions localized along stable (the so called "tube" functions) and unstable POs ("scar" functions). Then, in Sec. IV we present the results that have been obtained and the corresponding discussion. Finally, in Sec. V we summarize the main conclusions of this work and the outlook for further research.

\section{SYSTEM}

In this section we briefly describe the characteristics of the dynamical system that we have chosen to study, i.e., the $\mathrm{LiNC} / \mathrm{LiCN}$ isomerizing molecule, which are relevant for this work. We first discuss the effective vibrational Hamiltonian and the potential energy surface of the system in Subsection II A. Then, Subsection II B is devoted to the discussion of the dynamical characteristics of the vibrations of this molecule. In particular, we examine the chaoticity of the system as a function of the energy using Poincare surfaces of section (SOS). Finally, we conclude the section by presenting in Subsection II C the bifurcation-continuation diagram of the most relevant POs of the system taking the excitation energy as parameter. These POs will be used later in the construction of a semiclassical basis set for the computation of the vibrational eigenstates of the molecule (see discussion in Sec. IV).

\section{A. Hamiltonian}

The system under study is the $\mathrm{LiNC} / \mathrm{LiCN}$ isomerizing molecule which has been extensively studied in the past, especially in connection with quantum chaos. ${ }^{9,10,12-17,19,42-45}$ This system exhibits a very floppy motion in the angular coordinate and, as a consequence, chaos sets in at very moderate values of the excitation energy.

The corresponding vibrational motion can be adequately modeled with the following rotationless $(J=0)$ Hamiltonian

$$
\mathcal{H}=\frac{P_{R}^{2}}{2 \mu_{1}}+\frac{P_{r}^{2}}{2 \mu_{2}}+\frac{1}{2}\left(\frac{1}{\mu_{1} R^{2}}+\frac{1}{\mu_{2} r^{2}}\right) P_{\vartheta}^{2}+\mathcal{V}(R, r, \vartheta)
$$

in Jacobi coordinates, where $R$ and $\vartheta$ describe the $\mathrm{Li}-\mathrm{CN}$ stretching and $\mathrm{Li}-\mathrm{C}-\mathrm{N}$ bending motions, respectively, as sketched in the bottom-left corner of Fig. 1, while $r$ accounts for the $\mathrm{C}-\mathrm{N}$ motion. The associated reduced masses are $\mu_{1}=m_{\mathrm{Li}} m_{\mathrm{CN}} / m_{\mathrm{LiCN}}$ and $\mu_{2}=m_{\mathrm{C}} m_{\mathrm{N}} / m_{\mathrm{CN}}$, with $m_{\mathrm{LiCN}}=m_{\mathrm{Li}}$ $+m_{\mathrm{C}}+m_{\mathrm{N}}$ and $m_{\mathrm{CN}}=m_{\mathrm{C}}+m_{\mathrm{N}}$. For all practical purposes, the motion in the $r$ coordinate plays no role due to the strength of the $\mathrm{C}-\mathrm{N}$ triple bound, as reported by some of us elsewhere. ${ }^{44}$ Thus, one can keep frozen the $r$ coordinate at its equilibrium value, $r_{e}=2.186$ a.u., since the associated frequency is very high, and then decouples very effectively from the rest of the modes in the molecule. ${ }^{44}$ Consequently, we can use the following equivalent two-degrees-of-freedom Hamiltonian

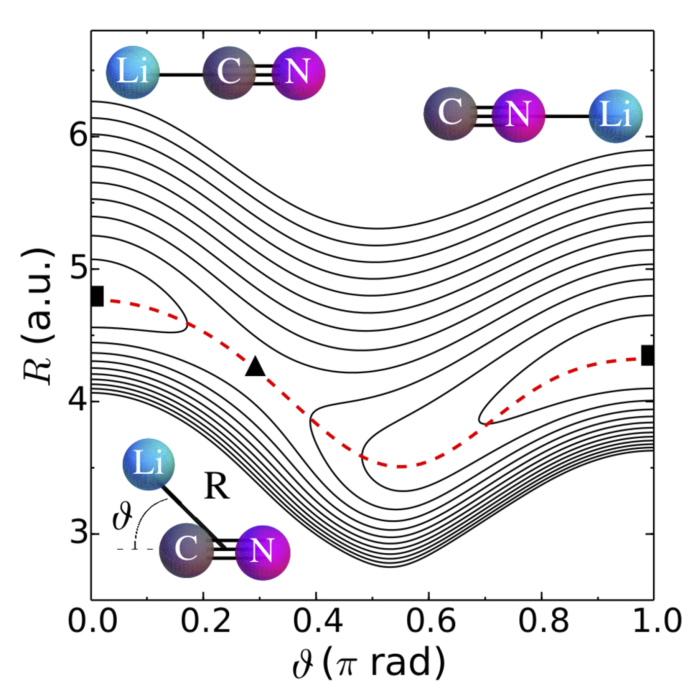

FIG. 1. Potential energy surface for the LiNC/LiCN molecular system represented as black contour lines separated $1000 \mathrm{~cm}^{-1}$ in the Jacobi coordinates, defined in the inset at the bottom-left corner. It presents two wells associated with the two existing stable linear isomers, LiNC and LiCN. Their positions are indicated with black squares, and their geometries are sketched in the insets at the top. The minimum energy path connecting these two wells passing through the saddle point, represented as a black triangle, has been plotted superimposed in a dashed red line. 


$$
\mathcal{H}=\frac{P_{R}^{2}}{2 \mu_{1}}+\frac{1}{2}\left(\frac{1}{\mu_{1} R^{2}}+\frac{1}{\mu_{2} r_{e}^{2}}\right) P_{\vartheta}^{2}+V(R, \vartheta),
$$

which still is able to retain all the complexity of the molecule under study, thus yielding at the same time results that are not only qualitative but also quantitative similar. ${ }^{44}$

The two-dimensional potential energy surface, $V(R, \vartheta)$, has been taken from the literature, ${ }^{46}$ and it is shown in Fig. 1 as a contours plot. Here, we have plotted for simplicity only the fundamental domain $\vartheta \in(0, \pi) \mathrm{rad}$ which results from the rotational symmetry. As can be seen, the potential presents two wells at $\vartheta=0$ and $\pi \mathrm{rad}$, respectively. They correspond to the two stable linear isomers, $\mathrm{LiCN}$ and $\mathrm{LiNC}$, existing for the molecule; their geometries are sketched at the top of the figure. These two isomers are separated by a modest energy barrier of only $E_{\mathrm{SP}} \sim 3454.0 \mathrm{~cm}^{-1}$ at the saddle point of the potential energy surface, where $(R, \vartheta)_{\mathrm{SP}}=(4.22$ a.u., $0.918 \mathrm{rad})$. The equilibrium point at the top of this barrier generates at higher energies an unstable PO that obviously plays a central role for the reactivity of the system. ${ }^{19}$ Finally, the minimum energy path (MEP) connecting the two potential minima has been plotted superimposed in the figure as a dashed red line.

\section{B. Chaos in the LiNC/LiCN system}

The dynamics of our model for the vibrations of the LiNC/LiCN molecule can be efficiently monitored by using Poincaré SOS, taking the MEP, $R_{e}(\vartheta)$, as the sectioning surface. $^{12}$ This choice maximizes the dynamical information obtained for the motion in the angular coordinate. However, this does not define an area preserving map satisfying the Liouville theorem. ${ }^{47}$ This inconvenience can be easily overcome by making the following canonical transformation:

$$
\begin{aligned}
\rho & =R-R_{e}(\vartheta), & \psi & =\vartheta, \\
P_{\rho} & =P_{R}, & P_{\psi} & =P_{\vartheta}+P_{R}\left[d R_{e}(\vartheta) / d \vartheta\right] .
\end{aligned}
$$

Some representative results, computed by numerically solving the equations of motion derived from Hamiltonian (2), using the Shampine and Gordon algorithm, ${ }^{48}$ for different values of the excitation energy, $E$, are shown in Fig. 2. As can be seen, the chaoticity of the system increases with the energy. At low energies, for example, $E=1000 \mathrm{~cm}^{-1}$ as chosen in Fig. 2(a), the vibrational motion takes place in the LiNC well and it is regular, being then confined in invariant tori. As higher energies are considered, e.g., panels (b) and (c), the invariant tori progressively start to break down, this paving the road for widespread chaotic motion, as dictated by the celebrated Kolmogorov-Arnold-Moser (KAM) theorem. ${ }^{49}$ Comparison of results in panels (b) and (c) clearly indicates that the dynamics in the LiNC well gets increasingly more chaotic as the excitation energy grows. In panel (c), which corresponds to an energy above the level of the less stable LiCN minimum well, motion also takes place in that region of the phase space. Also, a conspicuous accumulation of points next to the LiNC regular regions is observed. This is due to the existence of a cantorus, as thoroughly discussed in Ref. 50. At even higher energies, we end up being above the PES saddle energy, i.e., panel (d), the two isomer

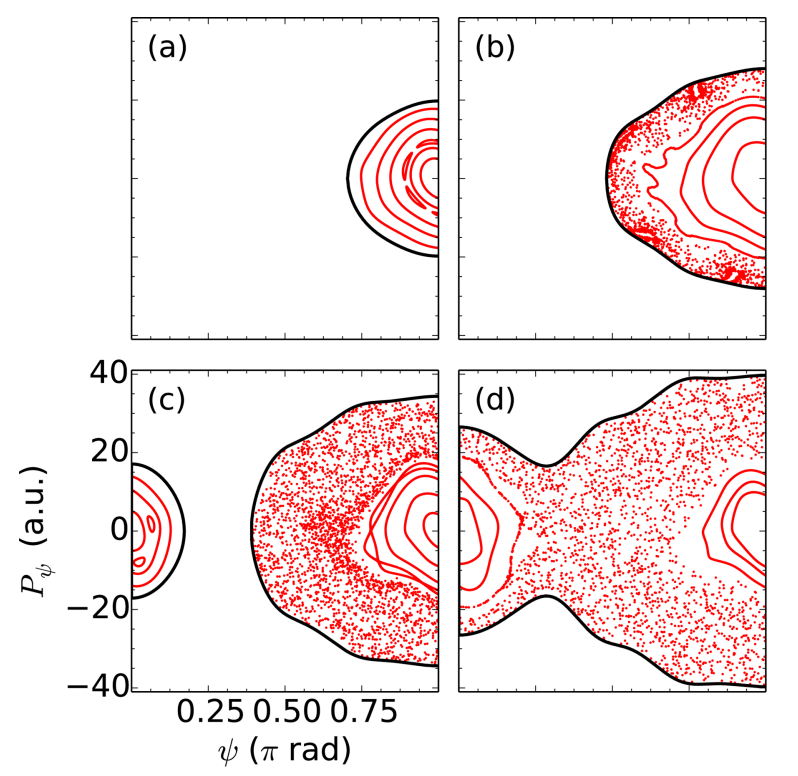

FIG. 2. Composite Poincaré surface of sections for the LiNC/LiCN vibrational dynamics computed along the minimum energy path shown in Fig. 1, i.e., $\rho=0$ [see Eq. (3)] at different values of the excitation energy: (a) $1000 \mathrm{~cm}^{-1}$, (b) $2000 \mathrm{~cm}^{-1}$, (c) $3000 \mathrm{~cm}^{-1}$, and (d) $4000 \mathrm{~cm}^{-1}$.

wells are then connected, this allowing classical isomerization dynamics.

\section{Periodic orbits for LiNC/LiCN and the bifurcation-continuation diagram}

Figure 3 shows the bifurcation-continuation diagram with the most relevant POs in the LiNC [panel (a)] and LiCN [panel (b)] wells, the transition state (TS) at the PES saddle, and also those "born" in the saddle-node or tangent bifurcation discussed in Ref. 50 [panel (c)]. The POs are characterized in this plots by the initial values of their stretch coordinate $R$ as a function of the energy. Thin blue and thick red lines indicate, respectively, the stability and instability of the corresponding orbits. As can be seen, the number of POs increases with energy due to the different bifurcations taking place. In panels (a)-(c) only the POs that are symmetric with respect to the $\vartheta=\pi$ and 0 rad lines, i.e., isomers $\mathrm{LiNC}$ and $\mathrm{LiCN}$, are considered. We have also highlighted in the figure with empty green circles the position of the quantized trajectories, i.e., the POs that fulfill the BS rule discussed below in Sec. III A 1. Moreover, those that will be used in our construction of a basis set for the system have been indicated with filled green circles (see Subsection III B). Notice also how the density of the states of the system increases with the excitation energy, as emphasized in the bottom panel (d), where the quantum energies of the system are represented.

The POs in Figs. 3(a) and 3(b) have been labeled as " $\mathrm{N} \mathrm{X}_{\mathrm{Y}-\mathrm{Z}}^{\mathrm{W}}$," $\mathrm{N}$ being an integer identifying the bifurcation at which they first appear (in all orbits considered $\mathrm{N}=1,2,3$ ). Letter $\mathrm{X}$ identifies the branch in the bifurcation diagram, being for librations or time-reversal POs $\mathrm{X}=\mathrm{A}$ associated with the upper branch and $\mathrm{X}=\mathrm{B}$ with the lower one; the rotations, i.e., POs that have no time-reversal symmetry and then correspond to both (upper and lower) branches, are labeled as $\mathrm{X}=\mathrm{AB} / \mathrm{BA}$. The $\mathrm{Y}$ subindex indicates the well where the PO 


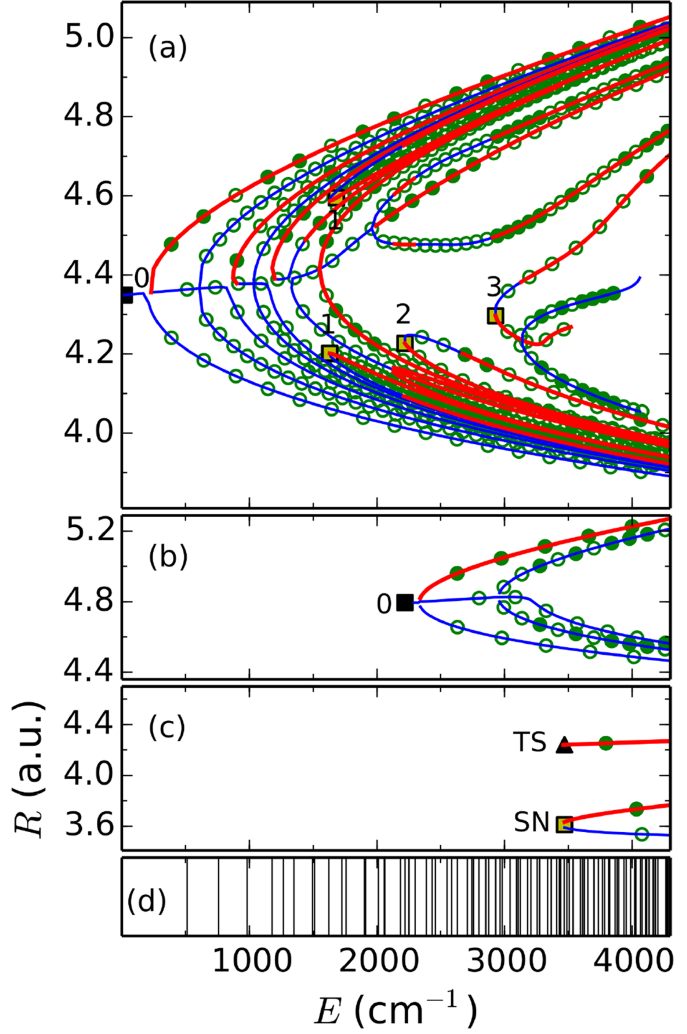

FIG. 3. Bifurcation-continuation diagram of periodic orbits (POs) for the $\mathrm{LiNC/LiCN}$ isomerizing system. Panels (a)-(c): Bifurcation-continuation diagrams for (a) POs in the LiNC isomer well, (b) same for LiCN, and (c) POs "born" both in the saddle-node bifurcation discussed in Ref. 50 (lower double red-blue line), and in the potential energy surface saddle (upper single red line). From top to bottom at the highest represented energy of $E=4300 \mathrm{~cm}^{-1}$, and in the notation used in Fig. 3 and throughout the text, (a) $1 \mathrm{~A}_{\pi-0}, 2 \mathrm{AB}_{\pi-0}, 3 \mathrm{~A}_{\pi-0}, 4 \mathrm{AB}_{\pi-0}, 5 \mathrm{~A}_{\pi-0}, 1 \mathrm{AB}_{\pi-1}, 1 \mathrm{BA}_{\pi-1}, 6 \mathrm{~A}_{\pi-0}$, $7 \mathrm{AB}_{\pi-0}, 8 \mathrm{AB}_{\pi-0}, 0_{\pi-0}, 8 \mathrm{AB}_{\pi-0}, 1 \mathrm{~A}_{\pi-3}, 2 \mathrm{AB}_{\pi-3}, 1 \mathrm{~B}_{\pi-3}, 2 \mathrm{AB}_{\pi-3}$, $2 \mathrm{AB}_{\pi-2}, 2 \mathrm{AB}_{\pi-2} 7 \mathrm{AB}_{\pi-0}, 9 \mathrm{AB}_{\pi-0}, 6 \mathrm{~B}_{\pi-0}, 9 \mathrm{AB}_{\pi-0}, 6 \mathrm{~B}_{\pi-0}, 9 \mathrm{AB}_{\pi-0}$, $1 \mathrm{BA}_{\pi-1}, 1 \mathrm{AB}_{\pi-1}, 5 \mathrm{~B}_{\pi-0}, 4 \mathrm{AB}_{\pi-0}, 3 \mathrm{~B}_{\pi-0}, 2 \mathrm{AB}_{\pi-0}$, and $1 \mathrm{~B}_{\pi-0}$, (b) $1 \mathrm{~A}_{0-0}$, $2 \mathrm{AB}_{0-0}, 0_{0-0}, 2 \mathrm{AB}_{0-0}$, and $1 \mathrm{~B}_{0-0}$, and (c) $\mathrm{TS}^{u}, \mathrm{SN}^{u}$, and $\mathrm{SN}^{s}$. Thin blue lines indicate stable POs, while unstable POs are referenced by thick red lines. The saddle point has been marked as black triangle, the two potential minima as black squares, and the lowest-lying bifurcation point of other important families of POs as yellow squares. The empty green circles represent the Bohr-Sommerfeld quantized energies determined by Eq. (6). The energies of the localized states selected for the construction of the basis set have been highlighted in filled circles (see discussion in Sec. IV). Panel (d): Quantum eigenenergies for the $\mathrm{LiNC/LiCN}$ system.

is located: $\mathrm{Y}=0$ for POs associated with the $\mathrm{LiCN}$ isomer, and $\mathrm{Y}=\pi$ for POs of the LiNC isomer. Subindex $\mathrm{Z}=0,1,2, \ldots$ is an integer indicating the bifurcation where the first $\mathrm{PO}$ appears. The stable/unstable character of the $\mathrm{PO}$ is indicated by $\mathrm{W}=\mathrm{s} / \mathrm{u}$ (for stable or unstable, respectively). The POs of panel (c) have been labelled as "TS" in the case of the trajectory located in the neighborhood of the TS or activated complex at the PES saddle point, and as "SN" ${ }^{s}$ " " $\mathrm{SN}^{u}$ ") for the case of the stable (unstable) POs "born" in the tangent bifurcation. ${ }^{50}$

All trajectories introduced in Fig. 3 are presented in Fig. 4 at a particular value of the energy, actually $E=3500 \mathrm{~cm}^{-1}$. In this figure, we have also included the POs corresponding to the stretch modes associated with purely vibrational motion of $R$ in both wells, which are always stable. We have labeled them as "S $\mathrm{S}_{\mathrm{Y}}$," where the subindex $\mathrm{Y}$ indicates again the well where the PO is localized ( $\mathrm{Y}=0$ for $\mathrm{LiCN}$ and $\pi$ for $\mathrm{LiNC}$ ).
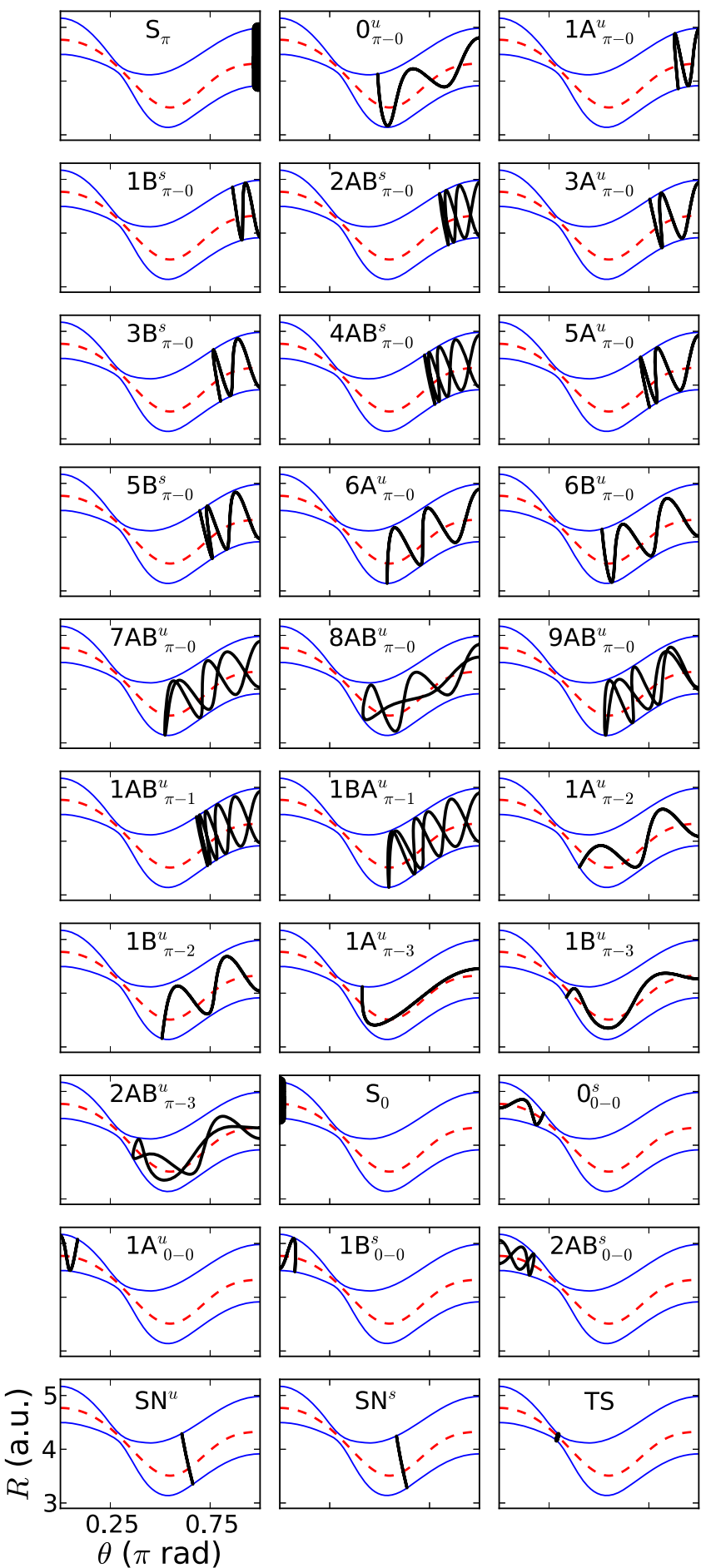

FIG. 4. Periodic orbits (black thick lines) of LiNC/LiCN molecular system included in the bifurcation-continuation diagram of Fig. 3. The minimum energy path and the equipotential lines at $3500 \mathrm{~cm}^{-1}$ have been superimposed in dashed red and continuous blue lines, respectively.

\section{METHOD}

In this section we describe the method that we have developed for the construction of an efficient semiclassical basis set. The section is divided in two parts. First, we describe in Subsection III A how to compute localized wave functions along POs. Second, in Subsection III B we discuss how the previous localized wave functions are selected for the construction of our basis set. 


\section{A. Computation of localized wave functions}

In this subsection, we briefly describe the method to construct localized wave functions along POs. For this purpose, we distinguish between two different kinds of states depending on whether the PO is stable or unstable: for stable POs, we will use the so-called "tube" wave functions described in Subsection III A 1, while for unstable POs we will construct the "scar" wave functions that are presented in Subsection III A 2. More details can be found in Refs. 19, 38, and 40.

\section{The "tube" wave functions}

Our "tube" wave functions are defined as

$$
\psi_{n}^{\text {tube }}(R, \vartheta)=\int_{0}^{T} d t e^{-i E_{n} t / \hbar} \phi(R, \vartheta, t),
$$

where $T$ is the period of the $\mathrm{PO}$ and $E_{n}$ the corresponding BS quantized energy (see discussion below). As can be seen, it consists of a time average of a suitably defined wave packet $\phi(R, \vartheta, t)$, whose dynamics is forced to stay in the neighborhood of the PO. This dynamics, given at time $t$ by the phase space point $\left(R_{t}, \vartheta_{t}, P_{R, t}, P_{\vartheta, t}\right)$, is assumed to be that of a frozen Gaussian ${ }^{51,52}$ centered on the trajectory as

$$
\begin{aligned}
\phi(R, \vartheta, t)= & \exp \left\{-\alpha_{R}\left(R-R_{t}\right)^{2}-\alpha_{\vartheta}\left(\vartheta-\vartheta_{t}\right)^{2}\right. \\
& \left.+\frac{i}{\hbar}\left[P_{R, t}\left(R-R_{t}\right)+P_{\vartheta, t}\left(\vartheta-\vartheta_{t}\right)\right]+i \gamma_{t}\right\} .
\end{aligned}
$$

Here, we take $\alpha_{R}=16.114$ a.u. $^{-2}$ and $\alpha_{\vartheta}=14.123 \mathrm{rad}^{-2}$ that approximately coincide with the "width" in configuration space of the LiNC quantum ground state. The time function $\gamma_{t}=S_{t} / \hbar-\mu_{t} \pi / 2$ is the phase accumulated during the propagation, which is actually the sum of two terms: the first one of dynamical origin given by $S_{t} / \hbar=\int_{0}^{t} d \tau\left(P_{R, \tau} \dot{R}_{\tau}+P_{\vartheta, \tau} \dot{\vartheta}_{\tau}\right) / \hbar$, and the second contribution proportional to $\mu_{t}$, which equals the number of half turns that the neighbouring trajectories describe around the guiding PO. This second term, which is always more complicated to compute, can be evaluated by using a set of local coordinates along the PO and studying the time evolution of the corresponding transversal stability matrix. ${ }^{53}$ It should be noticed that $\mu_{t}$ is not a canonical invariant, and as a result its magnitude depends on the definition chosen for the angle swept by the manifolds. Very often, only the value of this magnitude after a full period of the PO, $\mu_{T}$, usually known as the winding number is needed. In this case, the function is canonically invariant and is equal, for unstable POs, to the Maslov index ${ }^{54}$ appearing in Gutzwiller's trace formula. ${ }^{55,56}$ More importantly, the required phase becomes much easier to calculate, since it is simply equal to $\pi / 2$ times the number of turning points plus self-conjugated points in the PO.

In order to maximize the localization along the PO, the tube functions are defined at the energies, $E_{n}$, fulfilling the BS quantization rule

$$
\gamma=\frac{S\left(E_{n}\right)}{\hbar}-\mu \frac{\pi}{2}=2 \pi n, \quad n=0,1,2, \ldots,
$$

where $n$ is an integer number giving the number of nodes in the wave function along the PO, and $\gamma, S$, and $\mu$ being defined over one period of the PO, i.e., $\gamma=\gamma_{T}, S=S_{T}$, and $\mu=\mu_{T}$.

Notice that many orbits of Fig. 4 are symmetric with respect to $\vartheta=0$ or $\vartheta=\pi \mathrm{rad}$, while the considered wave functions are symmetric with respect to these values. This means that the tube functions associated with symmetric POs have an even number of excitations, i.e., $n$ is even. Thus, in order to simplify notation, the $n$ number used to identify these tube functions equals half the number of excitations.

\section{The "scar" wave functions}

The tube functions introduced in Eq. (4) can be constructed both over stable or unstable POs. However, in the latter case it is convenient to introduce an improvement by defining what we call "scar" functions which incorporate short time dynamical information on the homoclinic structure of the PO invariant manifolds. ${ }^{35}$

These scar functions are computed by first propagating the corresponding tube wave functions and then performing a finite-time Fourier transformation at the BS quantized energies in the following way:

$$
\begin{aligned}
& \psi_{n}^{\mathrm{scar}}(R, \vartheta)= \\
& \quad \int_{-T_{E}}^{+T_{E}} d t \cos \left(\frac{\pi t}{2 T_{E}}\right) e^{-i\left(\hat{\mathcal{H}}-E_{n}\right) t / \hbar} \psi_{n}^{\mathrm{tube}}(R, \vartheta),
\end{aligned}
$$

where

$$
T_{E}=\frac{1}{2 \lambda} \ln \left(\frac{A}{\hbar}\right)
$$

is the so-called Ehrenfest time, which can be only defined for unstable POs and depends on two parameters: the stability exponent of the $\mathrm{PO},{ }^{47} \lambda$, and the area of a characteristic SOS, $A$. This time can be (semiclassically) understood as the lapse of time that a Gaussian wave packet needs to spread over this characteristic Poincare SOS area of the system. Also, a cosine window is used in the definition (7) in order to minimize the dispersion in energy of the scar functions. ${ }^{57}$ Among other methods, wavelets provide an efficient method to perform the time evolution appearing in Eq. (7), with a precision of at least six decimal places. ${ }^{58}$

Figure 5 shows some examples of very highly excited scar functions along the quantized unstable POs $3 \mathrm{~A}^{u}$ of Fig. 4 , corresponding to the quantum numbers $n=14-19$ and BS energies between $3091.48 \mathrm{~cm}^{-1}$ and $3982.40 \mathrm{~cm}^{-1}$. In all examples

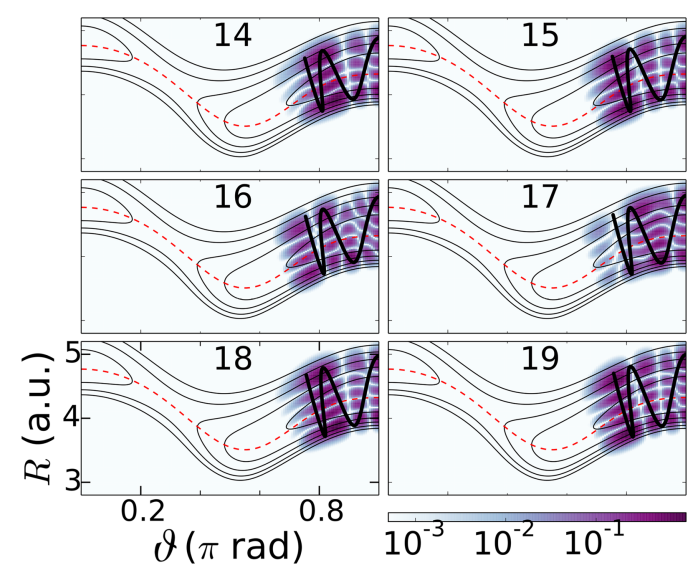

FIG. 5. Some examples of scar functions (5) for LiNC/LiCN. The unstable periodic orbit $3 \mathrm{~A}^{u}$ (think black line), the minimum energy path (dashed red line), and the contour plots of the potential energy surface have been plotted superimposed. The number in the center of each panel gives the integer appearing in Bohr-Sommerfeld quantization rule (6). 
shown in the figure the characteristic area, $A$, appearing in Eq. (8) has been estimated as the integral $\int d R P_{R}$ computed along the line $\vartheta=\pi$ rad at the quantized excitation energy, since the PO lives in the vicinity of that region. All these functions, as well as any other throughout the paper, have been computed by setting $\hbar=1$ a.u. As can be seen, the probability density is not well localized over the PO because of the complex topology of the trajectory, this somehow reducing the scarring phenomenon due to the quantum dynamics implied by finite $\hbar$. Also, notice that these functions are both excited in the $R$ and $\vartheta$ directions, and seem to have a rather simple pattern. ${ }^{19}$ Consequently, one can easily assign quantum numbers accounting for the number of excitations (or nodes) in each direction. For example, the scar functions shown at the top row of Fig. 5, labeled as 14 and 15, correspond to states with 3 excitations in each direction, and then $\left(n_{R}, n_{\vartheta}\right)=(3,3)$. The two scar functions presented in the middle row have different quantum numbers: while the one on the left (labeled as (16)) has $\left(n_{R}, n_{\vartheta}\right)=(4,3)$, the one on the right $(17)$ corresponds to $\left(n_{R}, n_{\vartheta}\right)=(2,4)$. Finally, the scar functions $((18)$ and $(19))$ shown in the bottom row are associated with $\left(n_{R}, n_{\vartheta}\right)=(2,4)$ and $\left(n_{R}, n_{\vartheta}\right)=(3,4)$, respectively. Nevertheless, it is simpler to label these functions as we have done by counting the number of nodes that they have along the (desymmetrized) PO. This number $n=14-19$, which has also been shown in each panel, equals the integer fulfilling the BS rule (6), for $\mu=16$.

\section{B. Selective Gram-Schmidt method (GSM) for the construction of the basis set}

As energy increases, the exponential proliferation of classical POs in floppy molecules leads to a dramatic increment in the number of quantized POs over which our localized wave functions can be defined. Thus, a selective procedure for the best suited localized states must be developed in order to construct an efficient basis set for the computation of vibrational states of this kind of systems that keeps the eigenvalue problem at moderate sizes. Afterwards, the Hamiltonian matrix associated with Eq. (2) can be computed, and then diagonalized using standard procedures.

This subsection is divided in two parts. First, Subsection III B 1 describes the algorithm developed for the construction of our basis set, which is called selective Gram-Schmidt method (SGSM). Second, we discuss in Subsection III B 2 the procedure that we have developed for presenting our results in a way that provides a clear physical insight into them.

\section{Definition of the basis set}

To define our basis set, we have generalized the usual Gram-Schmidt method (GSM), ${ }^{59}$ and developed a new selective Gram-Schmidt method (SGSM). This SGSM is the second pillar of our method, and it is able to choose a basis set of linearly independent functions in a vectorial space from a larger (overcomplete) set of functions that can be used to efficiently compute the chaotic eigenfunctions of our system. ${ }^{40}$

The SGSM starts from an initial set of $N$ localized (tube and scar) functions, $\left|\psi_{j}^{(0)}\right\rangle$, from which the procedure selects the minimum number of them, $N_{b} \leq N$, necessary to adequately describe the Hilbert space defined by the eigenfunctions whose energies are contained in a given energy window, that is, the SGSM defines a basis set in that window. The elements of this basis set $\left|\psi_{j_{i}}^{(0)}\right\rangle$, where subindex $i$ orders the elements according to their semiclassical relevance (see discussion below), are automatically selected with the aid of the conventional GSM. Thus, associated with the basis $\left|\psi_{j_{i}}^{(0)}\right\rangle$, we construct an auxiliary basis $\left|\varphi_{i}\right\rangle$, formed by the orthogonalization of $\left|\psi_{j_{i}}^{(0)}\right\rangle$. For example, if we set

$$
\left|\varphi_{1}\right\rangle=\left|\psi_{j_{1}}^{(0)}\right\rangle
$$

then a second auxiliary function $\left|\varphi_{2}\right\rangle$ is given by

$$
\left|\varphi_{2}\right\rangle=\frac{\left|\psi_{j_{2}}^{(1)}\right\rangle}{\left|\psi_{j_{2}}^{(1)}\right|}
$$

where $j_{2} \neq j_{1}$ and

$$
\left|\psi_{j_{2}}^{(1)}\right\rangle=\left|\psi_{j_{2}}^{(0)}\right\rangle-\left\langle\varphi_{1} \mid \psi_{j_{2}}^{(0)}\right\rangle\left|\psi_{j_{2}}^{(0)}\right\rangle
$$

and so on.

In our SGSM method, the selection procedure of the basis functions with a given symmetry for the calculation of the eigenenergies, $E$, up to a given energy

$$
E<E_{\text {ref }}
$$

is done automatically by using a definite set of rules, which are based on a selection parameter, $\eta$. For a given localized function $\eta$ is defined as

$$
\eta_{j}=\rho_{j}\left[\sigma_{j}^{2}+\left(\delta E_{j}\right)^{2}\right]^{1 / 2}
$$

This parameter depends on three terms. First, it depends on the density of states, $\rho_{j}$, at the quantization BS energy $E_{j}$, which is only relevant when the energy window is large. Second, it also depends on the tube/scar function's dispersion, given by

$$
\sigma_{j}=\sqrt{\left\langle\psi_{j}^{(0)}\left|\hat{\mathcal{H}}^{2}\right| \psi_{j}^{(0)}\right\rangle-\left\langle\psi_{j}^{(0)}|\hat{\mathcal{H}}| \psi_{j}^{(0)}\right\rangle^{2}},
$$

where $\hat{\mathcal{H}}$ is the quantum version of the classical Hamiltonian (2). Third, $\eta$ depends on a new parameter, $\delta E_{j}$, defined as

$$
\delta E_{j}= \begin{cases}0, & \text { if } E_{j} \leq E_{\mathrm{ref}} \\ E_{j}-E_{\mathrm{ref}}, & \text { if } E_{j}>E_{\mathrm{ref}}\end{cases}
$$

The function $\delta E_{j}$ is included in Eq. (10) in order to improve the numerical accuracy by reducing boundary effects. When large energy windows are considered, $\delta E_{j}$ has a small influence on the results, and then it can even be neglected. It is thus clear that the parameter $\eta$ introduced in Eq. (10) can be also defined using other criteria that account, for example, for the stability or the period of the POs. ${ }^{40}$ In this work, however, all these coefficients have been dropped out for simplicity. On the other hand, this has been done because we want to use a single selection parameter for all orbits, no matter if they are stable or unstable. Recall that the stability exponent is complex for stable POs, and then $\eta$ would no longer be real. On the other hand, the inclusion of the period in Eq. (10), as done in Ref. 40, renders less accurate results. This last result is a consequence of the barriers existing in the LiNC/LiCN system, which confine the POs in certain regions of the phase space. At low energies, this confinement is caused by the invariant tori. At higher energies, the dynamical barrier close to $\vartheta=0.611 \mathrm{rad}$ [see accumulation of points next to the LiNC regular region 
in Fig. 2(b)] acts as an effective quantum separatrix in the phase space. ${ }^{19}$ Furthermore, we also have the PES barrier separating the two isomers. On the contrary, in generic highly chaotic systems the unstable POs densely cover the system phase space.

The SGSM is then defined, in an algorithmic way, as follows:

- 0. With the method described in Subsection III A, we compute all the localized states, $\left|\psi_{j}^{(0)}\right\rangle$, whose BS quantized energies, $E_{j}$, fulfill Eq. (6) for the POs shown in Fig. 3(c), and Eq. (6) for the POs in Figs. 3(a) and 3(b) (cf. also Fig. 4), and are contained at the same time in the enlarged energy window defined by

$$
E_{j}<E_{\text {ref }}+2 \sigma_{j},
$$

where the term $2 \sigma_{j}$ is introduced to reduce border effects, being $\sigma_{j}$ given by Eq. (11). For the stable POs, normalized tube functions are computed, whereas for the unstable ones the scar functions are constructed. This is the most time demanding step of our procedure. It should be remarked here that for the system under study, similar results would be obtained using solely the tube wave functions. Moreover, they are also adequate for systems with a higher degree of chaoticity. ${ }^{40}$ However, we have decided to use the scar wave functions over the unstable POs as they have a lower dispersion in energy, rendering thus slightly better results. Let us finally remark that it can be a priori expected that the overlap of the tube and scar functions outside the enlarged window (13) with the desired system eigenfunctions is negligible, due to the fact that they were constructed minimizing their energy dispersion.

- 1. From the initial set of localized functions, $\left|\psi_{j}^{(0)}\right\rangle$, we select a smaller number of them, $N_{b} \leq N$, forming a basis set that is optimal for our purposes, as the number of accurately computed eigenfunctions scales linearly with $N_{b}$. Notice that the number of tube and scar functions calculated for this purpose, $N$, should always be greater than or equal to

$$
N_{b}=N_{\mathrm{sc}}\left(E_{r e f}+2 \sigma_{\mathrm{sc}}\right)+c_{b} \sigma_{\mathrm{sc}} \rho,
$$

where $N_{\mathrm{sc}}(E)$ and $\sigma_{\mathrm{sc}}$ are, respectively, semiclassical approximations to the number of states with an energy smaller than $E$ and to the scar function dispersion, ${ }^{57}$ and the term $c_{b} \sigma_{\mathrm{sc}} \rho, c_{b}$ being an adjustable parameter ensuring that the number of localized states is large enough to construct the basis; this parameter will be set equal to $c_{b}=6$ in Subsection IV A. If $N<N_{b}$, more (longer) POs, and consequently more localized functions, need to be included in the basis at this step, as described in step 0 .

The first element of our basis set is the tube or scar function with the smallest $\eta_{j}$ value

$$
\left|\varphi_{1}\right\rangle=\left|\psi_{j_{1}}^{(0)}\right\rangle, \quad \text { with } \frac{1}{\eta_{j_{1}}}=\max \left\{\frac{1}{\eta_{j}}\right\} .
$$

According to Eq. (10), this choice gives priority to the wave functions which are more localized in energy.
- 2.a. The remaining localized functions are then orthogonalized to $\left|\psi_{j_{1}}^{(0)}\right\rangle$ as

$$
\left|\psi_{j}^{(1)}\right\rangle=\left|\psi_{j}^{(0)}\right\rangle-\left\langle\varphi_{1} \mid \psi_{j}^{(0)}\right\rangle\left|\varphi_{1}\right\rangle, \quad j \neq j_{1} .
$$

- 2.b. The second element of the basis set is $\left|\psi_{j_{2}}^{(0)}\right\rangle$, where the index $j_{2}\left(j_{2} \neq j_{1}\right)$ satisfies

$$
\frac{\left|\psi_{j_{2}}^{(1)}\right|^{2}}{\eta_{j_{2}}}=\max \left\{\frac{\left|\psi_{j}^{(1)}\right|^{2}}{\eta_{j}}\right\}_{j \neq j_{1}},
$$

where the norm in the numerator has been introduced in order to make the basis set elements as different as possible between them. Indeed, notice that after the orthogonalization of Eq. (16) the more similar $\left|\psi_{j}^{(0)}\right\rangle_{j \neq j_{1}}$ is to $\left|\varphi_{1}\right\rangle$, the smaller the norm of function $\left|\psi_{j}^{(1)}\right|_{j \neq j_{1}}$ is. Then the auxiliary function $\left|\varphi_{2}\right\rangle$ is computed as

$$
\left|\varphi_{2}\right\rangle=\frac{\left|\psi_{j_{2}}^{(1)}\right\rangle}{\left|\psi_{j_{2}}^{(1)}\right|} .
$$

The previous steps, 2.a and 2.b, are repeated for all the remaining elements in the initial basis set of localized (tube and scar) functions, in such a way that the $n$th step in the procedure is defined as follows:

- n.a. New functions are obtained by orthogonalization to the auxiliary function in the previous step, $\left|\varphi_{n-1}\right\rangle$,

$$
\begin{aligned}
\left|\psi_{j}^{(n-1)}\right\rangle & =\left|\psi_{j}^{(n-2)}\right\rangle-\left\langle\varphi_{n-1} \mid \psi_{j}^{(n-2)}\right\rangle\left|\varphi_{n-1}\right\rangle, \\
j & \neq j_{1}, j_{2}, \ldots, j_{n-1} .
\end{aligned}
$$

Let us remark that the functions $\left|\psi_{j}^{(n-1)}\right\rangle$ are not only orthogonal to $\left|\varphi_{n-1}\right\rangle$ but also to all the already selected functions $\left|\varphi_{n-2}\right\rangle,\left|\varphi_{n-3}\right\rangle, \ldots,\left|\varphi_{2}\right\rangle,\left|\varphi_{1}\right\rangle$ because of the $n-2$ previous orthogonalizations.

- n.b. The $n$th basis element is $\left|\psi_{j_{n}}^{(0)}\right\rangle$, where the $j_{n}$ index satisfies

$$
\frac{\left|\psi_{j_{n}}^{(n-1)}\right|^{2}}{\eta_{j_{n}}}=\max \left\{\frac{\left|\psi_{j}^{(n-1)}\right|^{2}}{\eta_{j}}\right\}_{j \neq j_{1}, j_{2}, \ldots, j_{n-1}},
$$

and the next auxiliary function is constructed according to

$$
\left|\varphi_{n}\right\rangle=\frac{\left|\psi_{j_{n}}^{(n-1)}\right\rangle}{\left|\psi_{j_{n}}^{(n-1)}\right|} .
$$

- The procedure finishes when the number of selected elements in the basis set equals $N_{b}$ given by Eq. (14).

Afterwards, the corresponding Hamiltonian matrix is computed in the basis set of localized functions, or alternatively in the equivalent basis set of auxiliary functions. Diagonalization using standard routines ${ }^{60}$ finally renders $N_{b}$ eigenstates in the energy window defined in Eq. (9). Notice that while the (original) localized wave functions define a basis set that is nonorthogonal, and then a generalized eigenvalue problem should be solved, the basis set of the auxiliary functions is orthogonal, requiring then the solution of the standard eigenvalue problem. Recall that the computation time in both cases remains moderate when basis sets 
of dozens or hundreds of elements are used, but the generalized eigenvalue problem is, in general, much more demanding than the standard one when much larger basis sets are considered.

\section{Local representation}

To get a useful representation of the results obtained in our localized basis set construction procedure, a local representation should be used, in which each single eigenfunction is reconstructed as

$$
|N\rangle=\sum_{j=1}^{N_{b}} C_{N j}\left|\varphi_{j}^{\mathrm{loc}}\right\rangle,
$$

being $C_{N j}=\left\langle\varphi_{j}^{\mathrm{loc}} \mid N\right\rangle$, and $\sum_{j=1}^{N_{b}}\left|C_{N j}\right|^{2}=1$. The procedure to compute the functions $\varphi_{j}^{\text {loc }}$ is also based on the GSM, but in this case we give priority to those localized (tube/scar) wave functions with larger localization intensities, i.e., with a larger overlap with the eigenfunction $|N\rangle$. For this purpose, we proceed as follows:

- 1. The first element of the local representation is taken as the localized state, $\left|\psi_{j}^{(0)}\right\rangle$, with the largest localization intensity, which is defined as

$$
x_{j}^{(n)}=\left|\left\langle\psi_{j}^{(n)} \mid N\right\rangle\right|^{2} .
$$

Then,

$$
\left|\varphi_{1}^{\mathrm{loc}}\right\rangle=\left|\psi_{j_{1}}^{(0)}\right\rangle
$$

$x_{1} \equiv x_{j_{1}}^{(0)}=\max \left\{x_{j}^{(0)}\right\}$ being the largest localization intensity. This intensity provides valuable information on the localization of the $|N\rangle$ eigenfunction over the quantized orbit associated with $\left|\psi_{j_{1}}^{(0)}\right\rangle$.

- 2.a. For the identification of the second largest localization intensity, $x_{2}$, one must first orthogonalize the remaining localized states $\left|\psi_{j}^{(0)}\right\rangle$ to $\left|\varphi_{1}^{\text {loc }}\right\rangle$ in the following way:

$$
\left|\psi_{j}^{(1)}\right\rangle=\left|\psi_{j}^{(0)}\right\rangle-\left\langle\varphi_{1}^{\mathrm{loc}} \mid \psi_{j}^{(0)}\right\rangle\left|\varphi_{j}^{\mathrm{loc}}\right\rangle, \quad j \neq j_{1} .
$$

- 2.b. The second element of the local representation is defined as

$$
\left|\varphi_{2}^{\mathrm{loc}}\right\rangle=\frac{\left|\psi_{j_{2}}^{(1)}\right\rangle}{\left|\psi_{j_{2}}^{(1)}\right|},
$$

with $x_{2} \equiv x_{j_{2}}^{(1)}=\max \left\{x_{j}^{(1)}, j \neq j_{1}\right\}$.

Due to the orthogonalization in (26), the intensity $x_{2}$ cannot be directly related to the localization of the $|N\rangle$ eigenfunction over the PO, along which $\left|\psi_{j_{2}}^{(0)}\right\rangle$ is constructed. Nonetheless, the sum $x_{1}+x_{2}$ is the square of the modulus of the projection of $|N\rangle$ onto the subspace defined by $\left|\psi_{j_{1}}^{(0)}\right\rangle$ and $\left|\psi_{j_{2}}^{(0)}\right\rangle$.

The previous steps, 2.a and 2.b, are repeated until all $N_{b}$ auxiliary functions are computed, in such a way that the $n$th step is defined as follows:

- n.a. The remaining functions, $\left|\psi_{j}^{(n-2)}\right\rangle$, are orthogonalized to the last element of the local representation computed, $\left|\varphi_{n-1}^{\text {loc }}\right\rangle$, as

$$
\begin{aligned}
\left|\psi_{j}^{(n-1)}\right\rangle & =\left|\psi_{j}^{(n-2)}\right\rangle-\left\langle\varphi_{n-1}^{\mathrm{loc}} \mid \psi_{j}^{(n-2)}\right\rangle\left|\varphi_{n-1}^{\mathrm{loc}}\right\rangle, \\
j & \neq j_{1}, j_{2}, \ldots, j_{n-1} .
\end{aligned}
$$

- n.b. The $n$th element of the local representation is given by

$$
\left|\varphi_{n}^{\mathrm{loc}}\right\rangle=\frac{\left|\psi_{j_{n}}^{(n-1)}\right\rangle}{\left|\psi_{j_{n}}^{(n-1)}\right|},
$$

$$
\text { with } x_{n} \equiv x_{j_{n}}^{(n-1)}=\max \left\{x_{j}^{(n-1)}, j \neq j_{1}, j_{2}, \ldots, j_{n-1}\right\} .
$$

Recall here that the sum $x_{1}+x_{2}+\ldots+x_{n}$ is related to the projection of $|N\rangle$ onto the subspace defined by the localized functions $\left|\psi_{j_{1}}^{(0)}\right\rangle,\left|\psi_{j_{2}}^{(0)}\right\rangle, \ldots,\left|\psi_{j_{n-1}}^{(0)}\right\rangle$.

\section{RESULTS AND DISCUSSION}

In this section we present some results for the vibrational eigenstates of the floppy $\mathrm{LiNC} / \mathrm{LiCN}$ molecule obtained with our basis set of (semiclassical) functions localized on POs plus the corresponding discussion. The section is divided into four parts. First, in Subsection IV A, we give full details of our computational procedure, and demonstrate that each individual eigenfunction can be essentially reconstructed using a very small number of basis elements. Second, we present in Subsection IV B the localization intensities of the system eigenfunctions. Third, in Subsection IV C, we demonstrate the efficiency of our basis set by comparison with other standard approximations through the computation of the participation ratios. Finally, we conclude by presenting estimations of the error in the eigenenergies and the corresponding eigenfunctions in Subsection IV D.

\section{A. Spectrum of the LiNC/LiCN eigenfunctions in a basis set of functions localized along periodic orbits}

Using the method reported in Sec. III we have constructed a basis set formed by solely 90 elements, which is able to accurately describe the 66 low-lying eigenfunctions of the $\mathrm{LiNC} / \mathrm{LiCN}$ isomerizing system. The structure of all these eigenfunctions in our localized basis set is discussed in detail in the supplementary material.

The construction of our localized basis set is performed in the following three steps. First, we set in Eq. (14) the values of $E_{\text {ref }}=4100 \mathrm{~cm}^{-1}$ and $c_{b}=6$. Second, we calculate the quantization energies of each PO, which are shown with empty green circles in Fig. 3. Finally, we construct the tube functions for all these POs in the case they are stable, and scar functions for the unstable ones. This procedure renders a total number of 508 localized wave functions. From this whole set, our method has defined our final basis set by selecting the 90 best suited, 19 of them being tube functions and the remaining 71 scar functions. The BS energies of the selected states have been highlighted with filled green circles in Fig. 3. As it can be seen, the number of selected states increases with energy at a similar rate as the density of eigenfunctions. This can be clearly understood by comparing the number of states contained in a given energy window for panels (a) and (d). For example, if we take an energy window of $200 \mathrm{~cm}^{-1}$ in Fig. 3(d), we can 
see that it only includes one state if the energy is smaller than $1000 \mathrm{~cm}^{-1}$ (the level spacing for the three low-lying eigenenergies is $\sim 230$ a.u.), 2 if the energy is $\sim 1200 \mathrm{~cm}^{-1}, 3$ in the range $\sim 1500 \mathrm{~cm}^{-1}$, or 4 for energies $\sim 2000 \mathrm{~cm}^{-1}$ (see supplementary material for further information). Thus, as energy increases, the density of states increases accordingly. In Fig. 3(a) we can see that the BS energies of the localized functions that form our localized basis set follow a similar pattern: the number of selected states (filled green points) is very low at small energies and are quite separated, while they get closer and closer for higher energies. Likewise, a more detailed analysis of Fig. 3 (a) shows that the number of selected BS energies included in a window of 200 a.u. equals the number of eigenenergies just discussed.

As already stated in Subsection III A, the tube/scar functions have a very low dispersion in energy. ${ }^{19,30,35,36,38}$ One can then ask whether there is a similar relationship for the eigenfunctions computed in a basis set formed by these localized wave functions. The answer to this question is affirmative, as shown by the results in Fig. 6, where the spectra of some representative eigenfunctions in the local representation given by Eq. (22) are presented. In the picture, we have also indicated the most contributing localized states, via their quantized POs, to the reconstruction of the eigenfunctions $|53\rangle$ and $|65\rangle$ (red spectra). This will be discussed in more detail below (cf. Subsection IV C). As it can be seen, the spectrum of each eigenfunction is mainly concentrated around the corresponding eigenenergy, which is taken as the origin of the horizontal axis. Notice that the spectrum has been represented as a function of the difference between eigenenergy and BS quantized energy measured in units of the mean level spacing, $1 / \rho$, since
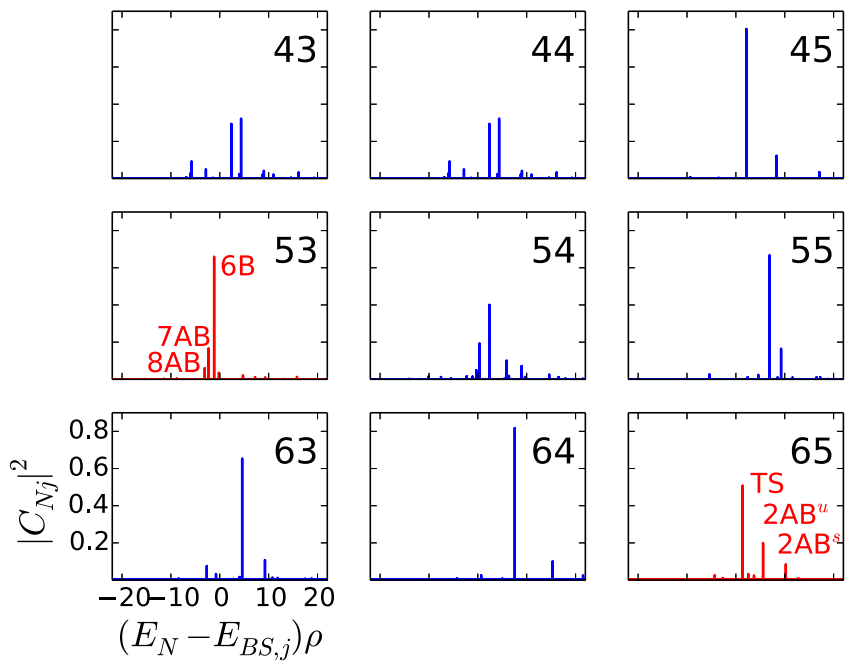

FIG. 6. Spectra of some representative LiNC/LiCN eigenfunctions in our set of localized basis functions. The vertical axis shows the squared of the coefficients of the eigenfunctions in the local representation given by Eq. (22). The horizontal axis used consists of the energy difference between the computed eigenenergy, $E_{N}$, and the Bohr-Sommerfeld quantized energy, $E_{B S, j}$, measured in units of the mean level spacing, $1 / \rho$. In the case of the eigenfunctions $|53\rangle$ and $|65\rangle$ (red) discussed in Subsection IV C, we have indicated which are the sticks associated with the tube/scar functions contributing the most, $\left(\left|6 \mathrm{~B}_{\pi-0}^{u}, 21\right\rangle,\left|7 \mathrm{AB}_{\pi-0}^{u}, 39\right\rangle\right.$, and $\left|8 \mathrm{AB}_{\pi-0}^{u}, 37\right\rangle$ for $|53\rangle ;\left|\mathrm{TS}^{u}\right\rangle,\left|2 \mathrm{AB}_{\pi-3}^{u}, 38\right\rangle$, and $\left|6 \mathrm{AB}_{0-0}^{u}, 6\right\rangle$ for $|65\rangle$ ), to the reconstruction (further details, see also Figs. 10 and 11 , and Sec. V). it provides a meaningful scaling. As already discussed in the previous paragraph, the density of states increases with the energy, and, as a consequence, the energy difference, i.e., the level spacing, between the eigenfunctions decreases. Thus, a comparison between two bare eigenenergies is not really very meaningful: one must also take into account the density of states in order to compare energy differences. For example, an energy difference of $10 \mathrm{~cm}^{-1}$ might be very small for the lowlying states, which have a mean level spacing of $\sim 230$ a.u., but being rather large for very excited states, where the number of eigenergies included in an window of $10 \mathrm{~cm}^{-1}$ is dramatically large. However, when the energy difference is measured in mean level spacing units by multiplying it by the density of states, it is very simple to say whether this relative energy difference is large or small: if it is larger than one, it must always be considered large, while it can be considered small if it is smaller than, at least, one half of the mean level spacing $(\leq 0.5)$.

Figure 7 shows (with empty red circles) the relative spectral dispersion of all computed LiNC/LiCN eigenfunctions, $\sigma_{r}$, defined as

$$
\sigma_{r}=\sigma_{N} \rho,
$$

where $\sigma_{N}$ is the dispersion of eigenfunction $|N\rangle$ in our semiclassical basis set. Then $\sigma_{r}$ measures the dispersion of eigenfunction $|N\rangle$ in mean level spacing units. In order to better identify the behaviour shown by this magnitude, we have also plotted superimposed its average value (filled red triangles), computed as a mobile mean of step 5 , which for a set of $M$ points $\left\{y_{1}, y_{2}, \ldots, y_{M}\right\}$ defined at the energies $\left\{E_{1}, E_{2}, \ldots, E_{M}\right\}$ is given by

$$
\bar{y}_{j}=\frac{1}{5} \sum_{i=1}^{5} y_{j+i}, \quad j=1,2, \ldots, M-5,
$$

being the corresponding (averaged) energy

$$
\bar{E}_{j}=\frac{1}{5} \sum_{i=1}^{5} E_{j+i}, \quad j=1,2, \ldots, M-5 .
$$

As can be seen, the average value of the dispersion increases with the energy, this being an indication of the necessity

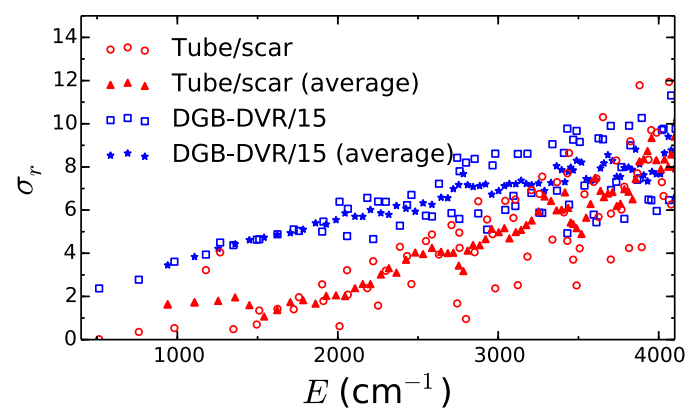

FIG. 7. Relative spectral dispersion (29) for the LiNC/LiCN eigenfunctions obtained with our basis set of localized function $v s$. the energy (red empty circles) and with a DGB-DVR basis set as defined in Ref. 9 (blue empty squares). In both cases, the average values, computed as a mobile mean of step 5 given by Eq. (30) for $y_{j} \equiv \sigma_{r j}$ and (31), has also been plotted superimposed (red filled triangles and blue filled stars, respectively). The DGB-DVR results have been divided by 15 in order that they are defined in the same range as our semiclassical results. 
of more basis elements for the reconstruction of the more excited eigenfunctions. Still, it should be remarked that the obtained values for dispersion of our basis set remain small compared to other standard methods. In order to demonstrate this assessment, we have superimposed in Fig. 7 the relative dispersion for a basis set formed by 345 basis elements defined by a combination of the Discrete Variable Representation (DVR) for the $\vartheta$ coordinate and a function representation of distributed Gaussian basis (DGB) in the radial coordinate $R{ }^{9}$ Recall that this kind of DGB-DVR basis sets have been extensively applied to the study of triatomic molecules such as $\mathrm{HCP},{ }^{61} \mathrm{HNC} / \mathrm{HCN},{ }^{62} \mathrm{H}_{2} \mathrm{O},{ }^{63} \mathrm{H}_{3}^{+},{ }^{64} \mathrm{KNC} / \mathrm{KCN},{ }^{20} \mathrm{SO}_{2},{ }^{65}$ $\mathrm{HO}_{2},{ }^{15}$ or the system under study, LiNC/LiCN. ${ }^{9,62}$ We have used 345 DGB-DVR basis elements which render computed eigenenergies with a precision of $0.1 \mathrm{~cm}^{-1}$. Notice that the DGB-DVR results have been divided over 15 in order that they are defined in the same range as our semiclassical calculations. Recall that the larger the DGB-DVR basis set, the larger the dispersion and the corresponding participation ratios (see discussion in Subsection IV C). As can be seen, both the bare relative dispersion (empty blue squares) and its average value (filled blue triangles) are between 15 and 30 times larger than the ones rendered by our localized basis set. Furthermore, as will be see below in Subsection IV D, this low dispersion of the eigenstates in our localized basis set, which is always smaller than 12 level spacing units, also reflects in a small value of the participation ratio, this fact further demonstrating the efficiency of our method.

\section{B. Localization intensities of the eigenfunctions}

In Fig. 8 we present, with empty red circles and empty blue squares, respectively, the two largest localization intensities $x_{1}$ and $x_{2}$ of the LiNC/LiCN eigenfunctions computed with our semiclassical basis set, as defined in Eq. (23). As can be seen, the fluctuation of both quantities is relatively large. Accordingly, in order to better identify their behaviours, we have also plotted superimposed in the figure the corresponding average values (full red triangles and full blue stars, respectively), computed as a mobile mean of step 5 (cf. Eqs. (30) and (31)). For the low-lying eigenfunctions, the intensity $x_{1}$

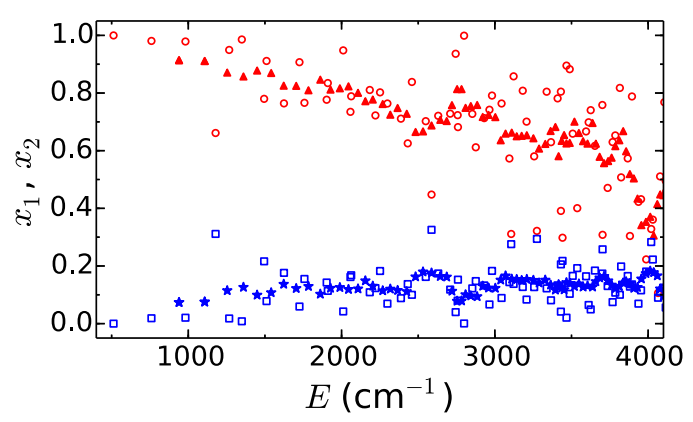

FIG. 8. Largest localization intensities $x_{1}$ (red empty circles) and $x_{2}$ (bottom blue empty squares) for the eigenfunctions of the $\mathrm{LiNC} / \mathrm{LiCN}$ system in our basis set of localized functions. The average, computed as a mobile mean of step 5, given by Eqs. (30) $y_{j} \equiv x_{1, j}, x_{2, j}$ and (31), is plotted superimposed with red full triangles and blue full stars, respectively. has a value close to 1 , thus indicating that those eigenfunctions are strongly concentrated over one single PO. Notice that the eigenfunctions that are highly localized over unstable POs, i.e., scar basis functions, correspond to "scars" of the system. ${ }^{6}$ When this happens, $x_{2}$ is smaller than its mean value, as $\Sigma_{j=1}^{N_{b}} x_{j}=1$. The average value of $x_{1}$, computed again as a mobile mean (cf. Eqs. (30) and (31)), decreases more or less monotonically with the energy. Meanwhile, the average value of $x_{2}$ increases up to $\sim 1400 \mathrm{~cm}^{-1}$, and then remains more or less constant and equal to $\bar{x}_{2} \approx 0.1$. Let us remark, nevertheless, that $x_{2}$ is by definition always smaller than $x_{1}$, and then it must also decrease for larger values of the energy although this is not noticeable in Fig. $8 .^{66}$

\section{Participation ratios and local representation of the eigenfunctions}

In order to have a more quantitative analysis of the quality of our basis set, we have also considered participation ratios, $R_{N}$, of the LiNC/LiCN eigenfunctions, $|N\rangle$, defined as [cf. Eq. (22)]

$$
R_{N}=\frac{\sum_{j=1}^{N_{b}} C_{N j}^{2}}{\sum_{j=1}^{N_{b}} C_{N j}^{4}} .
$$

When examining this magnitude, one has to take into account that the participation ratios defined in this way are bounded by two limiting cases. On one hand, the optimal basis set is always formed by the eigenfunctions system. In this case, all coefficients $C_{N j}$ appearing in Eq. (32) except one would vanish and, consequently, $R_{\min }=1$. On the other hand, the most illsuited basis set would be one where all the coefficients $C_{N j}$ were equal; in this case $R_{\max }=N_{b}$.

We present in Fig. 9 the participation ratios, $R_{N}$, for the LiNC/LiCN eigenfunctions computed with the basis set constructed with our procedure (red empty circles). As can be seen, most of the low-lying states have a value of the participation ratio close to (the optimal) one. This result is a consequence of the strong localization of these eigenfunctions along the POs considered for the basis construction. Thus, the overlap between our semiclassical basis elements and the eigenfunctions of the system becomes very large. As energy increases, more basis elements are necessary for the

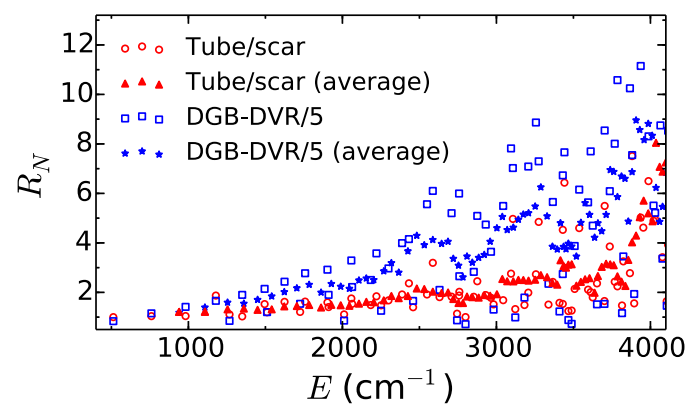

FIG. 9. Participation ratios for the LiNC/LiCN eigenfunctions obtained with our basis set of localized function $v s$. the energy (red empty circles) and with a DGB-DVR basis set as defined in Ref. 9 (blue empty squares). In both cases, the average values, computed as a mobile mean of step 5, given by Eqs. (30) for $y_{j} \equiv R_{j}$ and (31), have also been plotted superimposed (red filled triangles and blue filled stars, respectively). The DGB-DVR results have been divided by 5 in order to be defined in the same range as our semiclassical results. 
computation of the system eigenfunctions, and then the participation ratios increase accordingly. Although $R_{N}$ is seen to present large fluctuations with energy, especially for $E \gtrsim 3000$ $\mathrm{cm}^{-1}$, the results in Fig. 9 show that its average value increases quite smoothly. The dramatic increment of the participation ratio for $E \gtrsim 3800 \mathrm{~cm}^{-1}$ demonstrates the necessity of more basis elements, i.e., more POs are required. For a better observation of the tendency of the participation ratios, we have also plotted superimposed in the figure with red triangles their average values, computed again as a mobile mean of step 5 .

Let us remark that the participation ratios in our localized basis are much smaller than those obtained using other standard methods, like the results shown in Fig. 9 in empty blue squares, as well as than their average values presented in blue filled stars, which corresponds to the computation of the LiNC/LiCN eigenstates using a DGB-DVR basis set. Notice that these DGB-DVR results have been divided by 5 in order to be defined in the same range as the results rendered by the computations of our localized basis set.

Let us finally conclude this section by presenting two examples of the structure of the eigenfunctions obtained with our basis set. For this purpose we have selected the eigenstates $|53\rangle$ and $|65\rangle$ highlighted in red in Fig. 8. We present in Fig. 10 the results corresponding to the first case. Eigenfunction $|53\rangle$ has a participation ratio $R_{53}=2.16$, which implies that it can be essentially reconstructed by using only 2 or at most 3 basis elements. Moreover, it has a very irregular nodal pattern, something characteristic of classically chaotic systems, as shown to be the case here in Fig. 10(a). The most important contribution to this eigenfunction is given by the basis scar function $\left|6 \mathrm{~B}_{\pi-0}^{u}, 21\right\rangle$, which is shown in panel (b) of Fig. 10. Just by using this single basis function $65.9 \%$ of the (exact) eigenfunction $|53\rangle$ can be reconstructed, as shown in panel (e). When the scar function $\left|7 \mathrm{AB}_{\pi-0}^{u}, 39\right\rangle$ [see panel (c)] is added as a second element to the basis, $82.5 \%$ of the eigenfunction is reconstructed, see panel (f). Finally, augmenting the basis set with the scar function $\left|8 \mathrm{AB}_{\pi-0}^{u}, 37\right\rangle$ [see panel (d)] as the third element, $88.5 \%$ of the exact eigenfunction is recovered. We believe this result, i.e., by using only the 3 localized functions depicted in panels (b)-(d), one can obtain the state shown in panel $(\mathrm{g})$, which cannot be ascribed to any of the POs shown

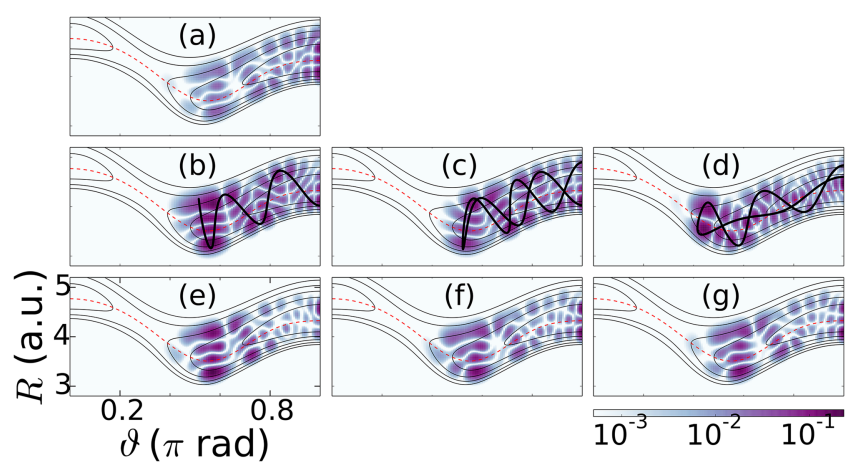

FIG. 10. Reconstruction of eigenfunction $|53\rangle$ of the $\mathrm{LiNC} / \mathrm{LiCN}$ system [shown in panel (a)]. The local representation is performed using the basis functions $\left|6 \mathrm{~B}_{\pi-0}^{u}, 21\right\rangle(\mathrm{b}),\left|7 \mathrm{AB}_{\pi-0}^{u}, 39\right\rangle(\mathrm{c})$, and $\left|8 \mathrm{AB}_{\pi-0}^{u}, 37\right\rangle(\mathrm{d})$. Using the wave function (b) one reconstructs the $65.9 \%$ of the exact eigenfunction (e); combining (b) and (c), one reconstructs $82.5 \%$ of it (f), and using (b), (c), and (d) $88.5 \%(\mathrm{~g})$. in Fig. 4, and it is quite impressive, this giving a clear idea of the quality and performance of our basis set construction method.

One last point is worth emphasizing in this discussion on the reconstruction of the eigenfunction $|53\rangle$ of $\mathrm{LiNC} / \mathrm{LiCN}$. The quantized energies corresponding to the basis elements which we have considered, i.e., those shown in panels (b)(d), lie quite close to the eigenenergy $E_{53}=3507.24 \mathrm{~cm}^{-1}$, as can also be seen in Fig. 6. Obviously, when increasing the number of basis elements this eigenfunction is more accurately reconstructed. For example, by including 6 basis elements, $95.1 \%$ of the exact eigenfunction is obtained; using 13 basis elements, 99.1\%; with 28 basis elements, $99.9 \%$, and using 39 basis elements, an impressive accuracy of $99.99 \%$ of the exact eigenfunction with an error of $0.66 \mathrm{~cm}^{-1}$ in the corresponding eigenenergy is obtained. In general, the accuracy of $99.9 \%$ in the reconstruction of the eigenfunctions is achieved by combination of less than 5 basis elements in the case of most of the low-lying states and around 25 of the 90 total basis elements for the most excited ones. Recall that the localized states selected in the reconstruction of these eigenfunctions are those with the BS quantized energies that lie closer to the considered eigenenergy. Let us recall here that we consider the exact results obtained with the 345 elements basis set needed in the calculation of Bačić and Light. ${ }^{9}$

Let us remark that in Fig. 10, the squared wave functions shown in panels (a)-(d) have been normalized such that their maximum value equals one. Contrary, the partially reconstructed eigenfunctions represented in panels $(\mathrm{e})-(\mathrm{g})$ have been normalized in such a way that the maximum value of the computed squared eigenfunction using the whole basis set of our localized wave functions equals one. As a consequence, the maximum value of the partially reconstructed eigenfunctions shown in panels (e)-(g) is always smaller than 1 . We have decided to present the results with these two different normalization criteria because then it is in general easier to visualize the contribution of each basis element to the eigenfunction reconstruction (cf. Fig. 11). Finally, notice that the scar function presented in panel (b) equals the reconstructed eigenfunction shown below in panel (e), the only difference between them being the normalization used. In Fig. 11 we show the results of a similar analysis performed for the structure of eigenfunction $|65\rangle$, which is the first isomerizing state of the system, i.e., having a significant proportion of the quantum density simultaneously localized in both isomer wells. This eigenfunction is shown in panel (a). It has a participation ratio equal to $R_{65}=4.60$, and the corresponding computed eigenenergy of $3826.84 \mathrm{~cm}^{-1}$ is $3.08 \mathrm{~cm}^{-1}$ smaller than in the DGB-DVR calculation taken as reference. Again, the eigenfunction is mostly reconstructed using a very small number of basis elements. Indeed, by just considering the scar function $\left|\mathrm{TS}^{u}, 0\right\rangle$ (b), $50.7 \%$ of the reference eigenfunction is recovered [see result in panel (e)]. Considering the scar functions in panels (b) and (c), one reconstructs $70.6 \%$ of the reference eigenfunction, as seen in panel (f). Finally, combining all basis functions shown in panels (b)-(d) and (h) $-(\mathrm{j})$, one gets the wave function shown in the panel $(\mathrm{m})$, which is very similar to the exact eigenfunction of panel (a), despite the fact that 

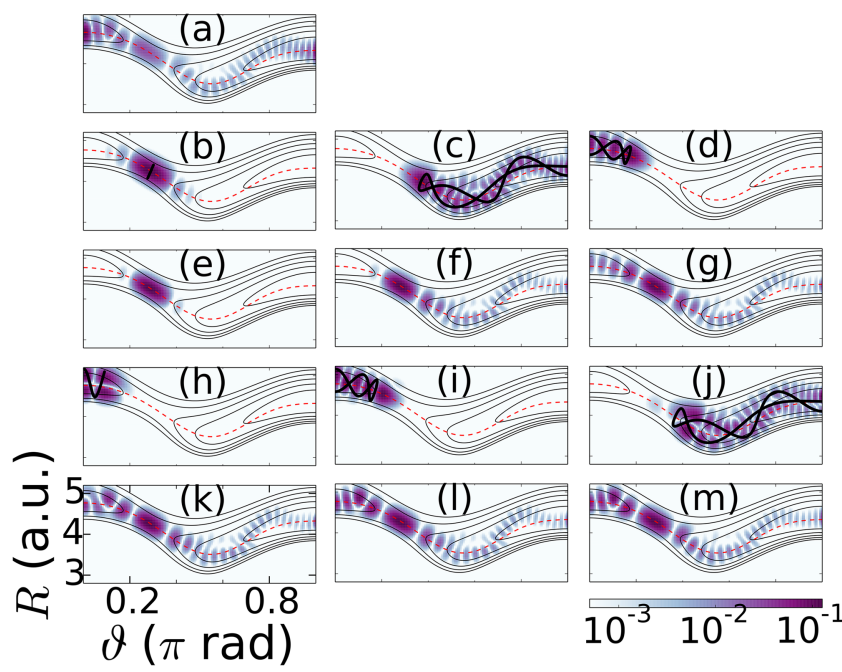

FIG. 11. Reconstruction of eigenfunction $|65\rangle$ of the LiNC/LiCN system [shown in panel (a)]. The local representation is performed using $\left|\mathrm{TS}^{u}, 0\right\rangle$ (b), $\left|2 \mathrm{AB}_{\pi-3}^{u}, 38\right\rangle(\mathrm{c})$, and $\left|2 \mathrm{AB}_{0-0}^{s}, 6\right\rangle(\mathrm{d}),\left|1 \mathrm{~A}_{0-0}^{u}, 3\right\rangle(\mathrm{h}),\left|2 \mathrm{AB}_{0-0}^{s}, 8\right\rangle$ (i), $\left|2 \mathrm{AB}_{\pi-0}^{u}, 39\right\rangle(\mathrm{j})$. The reconstruction process is shown in the remaining panels, where $50.7 \%$ (e), $70.6 \%$ (f), $78.9 \%$ (g), $82.2 \%$ (k), $84.7 \%$ (1) $87.0 \%$ (m) of the exact result is obtained.

it has been calculated using functions that are localized over nonisomerizing POs. Actually, the overlap between the exact eigenfunction and the approximate one computed using this, six elements, basis set equals an excellent $87.0 \%$. By using 12 basis elements, $95.3 \%$ of the exact eigenfunction is reconstructed, and by combination of 38 basis elements, $99.0 \%$. Recall that the localized (tube and scar) wave functions and the partially reconstructed eigenfunctions shown in Fig. 11 have been normalized using different criteria (see discussion on Fig. 10). For further information on the structure of all the 66 accurately computed eigenfunctions obtained with our localized basis set, see the supplementary material.

\section{Errors in the eigenenergies and the eigenfunctions}

Let us conclude this section by presenting the results rendered by our localized basis set. For this purpose, we compare the eigenenergies, $E$, and corresponding eigenfunctions, $|N\rangle$, with those taken as reference, $E^{\prime}$ and $\left|N^{\prime}\right\rangle$, which are obtained with the DGB-DVR method discussed at the end of Subsection IV A. Figure 12 shows the error in the eigenenergies measured in mean level spacing units, $\Delta E_{r}=\left|E-E^{\prime}\right| \rho$, (top red circles), and in the corresponding eigenfunctions, $1-\left\langle N^{\prime} \mid N\right\rangle^{2}$, (bottom blue asterisks), respectively, computed using our localized basis set as a function of the relative dispersion, $\sigma_{r}$, given by Eq. (29). As can be inferred from the figure and $a$ priori expected, both errors increase with the relative dispersion.

The black lines in Fig. 12 correspond to the upper bound for the errors in the energies and eigenfunctions of our vibrational states calculation given by

$$
\Delta E_{r} \leq \frac{4}{3} \sigma_{r}^{3 / 4}, \quad 1-\left\langle N^{\prime} \mid N\right\rangle^{2} \leq \sigma_{r},
$$

which indicates that the error in the eigenenergies scales as $\sigma_{r}^{3 / 4}$ with the relative dispersion, while that in the eigenfunctions does it linearly. Let us remark that Eq. (33) has been

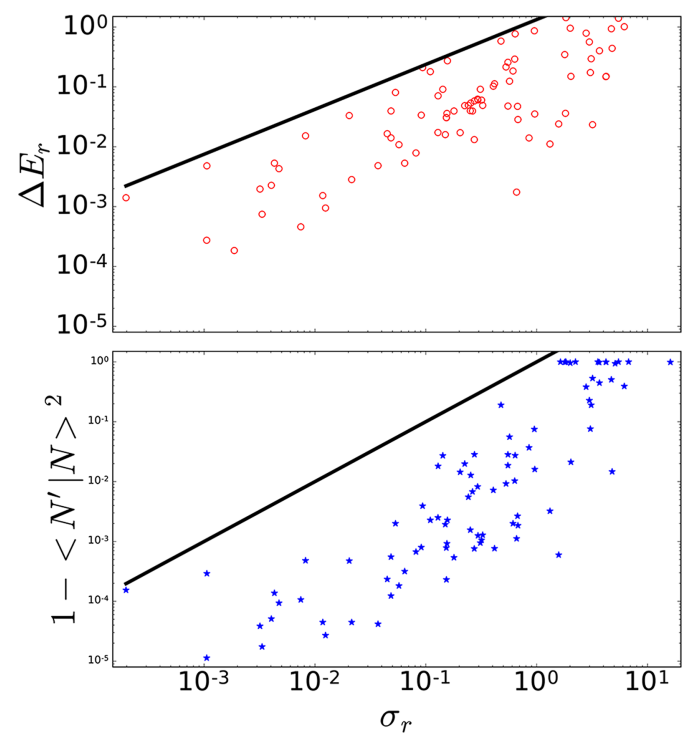

FIG. 12. Error in the eigenenergies (top red circles) and eigenfunctions (bottom blue asterisks) of the eigenstates using our localized basis set, estimated as described in Sec. IV D, as a function of the relative dispersion (29). The solid lines indicate the upper error bounds given by Eq. (33).

obtained heuristically, so one could equally define well the other (in general more complicated) functions to estimate the upper bounds. However, we have decided to use these expressions as they are extremely simple, and similar to those previously used by some of us in the study of other classically chaotic systems. ${ }^{40,57}$

Let us finally remark the usefulness of Eq. (33) as one can use them to know a priori the errors expected in the calculation of highly excited states ${ }^{40,57}$ by simply measuring the relative dispersion, which is very easy to calculate parameter.

\section{CONCLUSIONS AND OUTLOOK}

Summarizing, we have presented a method to efficiently compute the vibrational eigenstates of floppy molecular systems, in which the classical phase space contains regions of regular and irregular motion at the same energy. The method uses the so-called tube and scar wave functions, respectively, localized over stable and unstable POs, which then semiclassically account for these underlying classical structures of the system; this including short pieces of the invariant manifolds originated in the fixed point in the latter case. This method was originally introduced in Ref. 40, where it was applied to a highly chaotic system consisting of a homogeneous quartic coupled potential function. In this paper, we have extended that work by applying it to the study of a floppy molecule described by a realistic potential, namely, the $\mathrm{LiNC} / \mathrm{LiCN}$ isomerizing system. Using a basis set formed by 90 localized elements, we have accurately computed the 66 low-lying eigenenergies and the corresponding eigenfunctions of the system. More importantly, we have demonstrated that each eigenfunction is essentially reconstructed by a small number of basis elements, usually less than 5 in the energy range considered. Likewise, in order to demonstrate the efficiency of the method, a detailed discussion on the results has been performed, including an analysis of the structure of the eigenfunctions in terms of our 
efficient basis set, localization intensities, participation ratios, and also the errors of our computations, taking as reference the corresponding values as rendered by the DGB-DVR method of Bačić and Light. ${ }^{9}$

Finally, let us remark that the extension of our approach to the full three-degrees-of-freedom calculations of $\mathrm{LiNC} / \mathrm{LiCN}$ is straightforward, since it simply consists of making a direct product basis of the current functions and functions describing the third degree of freedom, $r$. However, the results reported by some of us in Ref. 44 indicate that significant changes in the conclusions of the present work should not be expected.

Regarding this point, it should be mentioned that the computational burden involved in a calculation for systems with more than two degrees of freedom will be only significantly increased, especially in systems with no symmetry, in the step of the identification of the required POs, a task that would then imply a search in more than one dimension. Also, the time propagation necessary for the construction of scar functions would require some more computational time. Still, and due to the small dispersion of these localized states, the size of the localized basis set will scale (approximately) linearly with the number of eigenfunctions of the system, which just increases following the Weyl law. ${ }^{4}$

\section{SUPPLEMENTARY MATERIAL}

See supplementary material for full details on the reconstruction of the 66 eigenenergies and eigenfunctions of the $\mathrm{LiNC} / \mathrm{LiCN}$ isomerizing system.

\section{ACKNOWLEDGMENTS}

We acknowledge financial support of the Spanish Ministry of Economy and Competitiveness (MINECO) under Contract Nos. MTM2012-39101 and MTM2015-63914-P and ICMAT Severo Ochoa under Contract No. SEV-2015-0554. We also thank Professor À. Jorba for having provided us the ShampineGordon routines used in our classical calculations.

${ }^{1}$ F. A. Berezin and M. Shubin, The Schrödinger Equation, Astrophysics and Space Science Library (Springer Netherlands, 1991), ISBN: 079231218X and 9780792312185 .

${ }^{2}$ L. Marchildon, Quantum Mechanics: From Basic Principles to Numerical Methods and Applications, Advanced Texts in Physics, 1st ed. (Springer-Verlag, Berlin, Heidelberg, 2002), ISBN: 978-3-642-07767-8 and 978-3-662-04750-7.

${ }^{3}$ G. C. Corey, J. W. Tromp, and D. Lemoine, Numerical Grid Methods and Their Application to Schrödingers Equation, NATO ASI Series Vol. 412, 1st ed. (Springer, Netherlands, 1993), ISBN: 978-90-481-4308-5 and 978-94-015-8240-7.

${ }^{4}$ M. Brack and R. K. Bhaduri, Semiclassical Physics, The Advanced Book Program (Addison-Wesley Publishing Company, Inc., Reading, Massachussetts, 1997), ISBN: 0-201-48351-3.

${ }^{5}$ M. C. Gutzwiller, Chaos in Classical and Quantum Mechanics, Interdisciplinary Applied Mathematics (Springer Science+Business Media New York, New York, 1990), ISBN: 978-1-4612-6970-0.

${ }^{6}$ E. J. Heller, Phys. Rev. Lett. 53, 1515 (1984).

${ }^{7}$ A. I. Shnirelman, Usp. Mat. Nauk 29, 181 (1974).

${ }^{8}$ Trends in Nano- and Micro-Cavities, edited by O. Kwon, B. Lee, and K. An (Bentham Science Publishers, 2011), ISBN: 9781608052363 and 1608052362.

${ }^{9}$ Z. Bačić and J. C. Light, J. Chem. Phys. 85, 4594 (1986).
${ }^{10}$ J. Tennyson, G. Brocks, and S. C. Farantos, Chem. Phys. 104, 399 (1986).

${ }^{11}$ S. C. Farantos and J. Tennyson, "Chaos in molecular systems?," in Stochasticity and Intramolecular Redistribution of Energy (Springer, Netherlands, Dordrecht, 1987), pp. 15-30, ISBN: 978-94-009-3837-3.

${ }^{12}$ R. Benito, F. Borondo, J.-H. Kim, B. Sumpter, and G. Ezra, Chem. Phys. Lett. 161, 60 (1989).

${ }^{13}$ J. R. Henderson and J. Tennyson, Mol. Phys. 69, 639 (1990).

${ }^{14}$ F. J. Arranz, F. Borondo, and R. M. Benito, J. Chem. Phys. 107, 2395 (1997); Phys. Rev. Lett. 80, 944 (1998).

${ }^{15}$ F. J. Arranz, L. Seidel, C. G. Giralda, R. M. Benito, and F. Borondo, Phys. Rev. E 82, 026201 (2010).

${ }^{16} \mathrm{~F}$. Borondo and R. M. Benito, in Nonlinear Dynamics and Fundamental Interactions, NATO Science Series Vol. 213, edited by F. Khanna and D. Matrasulov (Springer, Dordrecht, 2006), pp. 115-128.

${ }^{17}$ P. Benítez, J. C. Losada, R. M. Benito, and F. Borondo, "Analysis of the full vibrational dynamics of the $\mathrm{LiNC} / \mathrm{LiCN}$ molecular system," in Progress and Challenges in Dynamical Systems: Proceedings of the International Conference Dynamical Systems: 100 Years after Poincaré, September 2012, Gijón, Spain (Springer Berlin Heidelberg, Berlin, Heidelberg, 2013), pp. 77-88.

${ }^{18}$ H. Párraga, F. J. Arranz, R. M. Benito, and F. Borondo, J. Chem. Phys. 139, 194304 (2013).

${ }^{19}$ F. Revuelta, E. Vergini, R. M. Benito, and F. Borondo, "Scar functions, barriers for chemical reactivity, and vibrational basis sets," J. Phys. Chem. A 120, 4928 (2016).

${ }^{20}$ J. R. Henderson, H. A. Lam, and J. Tennyson, J. Chem. Soc., Faraday Trans. 88, 3287 (1992).

${ }^{21}$ G. Casati and B. Chirikov, Quantum Chaos: Between Order and Disorder (Cambridge University Press, 1995), ISBN: 052143291X, 9780521031660, 0521031664,9780521432917 , and 9780511599989.

${ }^{22}$ J. U. Nockel and A. D. Stone, Nature 385, 45 (1997).

${ }^{23}$ H. J. Stöckmann, Quantum Chaos: An Introduction, 1st ed. (Cambridge University, Cambridge, 2006), see https://cds.cern.ch/record/941387.

${ }^{24}$ C. Michel, V. Doya, O. Legrand, and F. Mortessagne, Phys. Rev. Lett. 99, 224101 (2007).

${ }^{25}$ S.-B. Lee, J.-H. Lee, J.-S. Chang, H.-J. Moon, S. W. Kim, and K. An, Phys. Rev. Lett. 88, 033903 (2002); H. Kwak, Y. Shin, S. Moon, S.-B. Lee, J. Yang, and K. An, Sci. Rep. 5, 9010 (2015).

${ }^{26}$ P. B. Wilkinson, T. M. Fromhold, L. Eaves, F. W. Sheard, N. Miura, and T. Takamasu, Nature 380, 608 (1996).

${ }^{27}$ L. Huang, Y.-C. Lai, D. K. Ferry, S. M. Goodnick, and R. Akis, Phys. Rev. Lett. 103, 054101 (2009); H. Xu, L. Huang, Y.-C. Lai, and C. Grebogi, ibid. 110, 064102 (2013).

${ }^{28}$ J. Larson, B. M. Anderson, and A. Altland, Phys. Rev. A 87, 013624 (2013).

${ }^{29}$ E. B. Bogomolny, Physica D 31, 169 (1988).

${ }^{30}$ G. G. de Polavieja, F. Borondo, and R. M. Benito, Phys. Rev. Lett. 73, 1613 (1994).

${ }^{31}$ M. V. Berry, Proc. R. Soc. A 243, 219 (1989).

${ }^{32}$ J. P. Keating and S. D. Prado, Proc. R. Soc. A 457, 1855 (2001).

${ }^{33}$ S. Tomsovic and E. J. Heller, Phys. Rev. Lett. 70, 1405 (1993); S. Tomsovic and J. H. Lefebvre, ibid. 79, 3629 (1997); D. A. Wisniacki, F. Borondo, E. Vergini, and R. M. Benito, Phys. Rev. E 63, 066220 (2001); D. A. Wisniacki, E. Vergini, R. M. Benito, and F. Borondo, ibid. 70, 035202 (2004); Phys. Rev. Lett. 94, 054101 (2005); 97, 094101 (2006).

${ }^{34}$ D. Wisniacki and G. G. Carlo, Phys. Rev. E 77, 045201 (2008); M. Novaes, J. M. Pedrosa, D. Wisniacki, G. G. Carlo, and J. P. Keating, ibid. 80, 035202 (2009).

${ }^{35}$ E. G. Vergini, J. Phys. A 33, 4709 (2000); E. G. Vergini and G. G. Carlo, ibid. 33, 4717 (2000).

${ }^{36}$ E. G. Vergini and G. G. Carlo, J. Phys. A 34, 4525 (2001).

${ }^{37}$ E. L. Sibert III, E. Vergini, R. M. Benito, and F. Borondo, New J. Phys. 10, 053016 (2008).

${ }^{38}$ F. Revuelta, E. G. Vergini, R. M. Benito, and F. Borondo, Phys. Rev. E 85, 026214 (2012).

${ }^{39}$ A. Vagov, H. Schomerus, and V. V. Zalipaev, Phys. Rev. E 80, 056202 (2009).

${ }^{40}$ F. Revuelta, R. M. Benito, F. Borondo, and E. Vergini, Phys. Rev. E 87, 042921 (2013).

${ }^{41}$ T. B. H. Skokos, Complex Hamiltonian Dynamics, Springer Series in Synergetics, Springer Complexity Vol. 10 (Springer, 2012), ISBN: 9783642273056 and $364227305 \mathrm{X}$, see http://gen.lib.rus.ec/book/index. php?md5=007AB157F53B453DE0DC214A51088E7B.

${ }^{42}$ P. L. García-Müller, F. Borondo, R. Hernandez, and R. M. Benito, Phys. Rev. Lett. 101, 178302 (2008). 
${ }^{43}$ G. E. Murgida, D. A. Wisniacki, P. I. Tamborenea, and F. Borondo, Chem. Phys. Lett. 496, 356 (2010).

${ }^{44}$ P. L. Garcia-Muller, R. Hernandez, R. M. Benito, and F. Borondo, J. Chem. Phys. 141, 074312 (2014).

${ }^{45}$ G. E. Murgida, F. J. Arranz, and F. Borondo, J. Chem. Phys. 143, 214305 (2015).

${ }^{46}$ R. Essers, J. Tennyson, and P. E. S. Wormer, Chem. Phys. Lett. 89, 223 (1982).

${ }^{47}$ A. J. Lichtenberg and M. A. Lieberman, "Regular and chaotic dynamics," in Applied Mathematical Sciences (Springer, New York, Berlin, Heidelberg, 2010), ISBN: 3-540-97745-7.

${ }^{48}$ L. F. Shampine and M. K. Gordon, Computer Solution of Ordinary Differential Equations: The Initial Value Problem (W. H. Freeman, USA, 1975), ISBN: 07-167-0461-7.

${ }^{49}$ V. I. Arnold, K. Vogtmann, and A. Weinstein, Mathematical Methods of Classical Mechanics, Graduate Texts in Mathematics (Springer-Verlag, New York, 1989), ISBN: 978-0-387-96890-2.

${ }^{50}$ F. Borondo, A. A. Zembekov, and R. M. Benito, Chem. Phys. Lett. 246, 421 (1995); J. Chem. Phys. 105, 5068 (1996); A. A. Zembekov, F. Borondo, and R. M. Benito, ibid. 107, 7934 (1997).

${ }^{51}$ E. J. Heller, J. Chem. Phys. 65, 4979 (1976).

${ }^{52}$ R. G. Littlejohn, Phys. Rep. 138, 193 (1986).

${ }^{53}$ B. Eckhardt and D. Wintgen, J. Phys. A 24, 4335 (1991).
${ }^{54}$ V. P. Maslov and M. V. Fedoriuk, "Semi-classical approximation in quantum mechanics," in Mathematical Physics and Applied Mathematics (D. Reidel Publishing Company, Dordrecht, Holland, Boston, USA, 1991), ISBN: 90-277-1219-0.

${ }^{55}$ S. C. Creagh, J. M. Robbins, and R. G. Littlejohn, Phys. Rev. A 42, 1907 (1990).

${ }^{56}$ J. M. Robbins, Nonlinearity 4, 343 (1991).

${ }^{57}$ E. G. Vergini, D. Schneider, and A. M. F. Rivas, J. Phys. A 41, 405102 (2008).

${ }^{58}$ D. K. Sparks and B. R. Johnson, J. Chem. Phys. 125, 114104 (2006).

${ }^{59}$ S. Lang, Algebra (Springer, 2002), ISBN: 978-1-4613-0041-0.

${ }^{60}$ W. H. Press and M. Metcalf, Numerical Recipes in Fortran 90: The Art of Parallel Scientific Computing: Volume 2 of Fortran Numerical Recipes, Fortran Numerical Recipes (Cambridge University Press, Cambridge, 1996), ISBN: 0-521-57440-4.

${ }^{61}$ F. J. Arranz, Z. S. Safi, R. M. Benito, and F. Borondo, Eur. Phys. J. D 60, 279 (2010).

${ }^{62}$ Z. Bačić, J. Chem. Phys. 95, 3456 (1991).

${ }^{63}$ Z. Bačić, D. Watt, and J. C. Light, J. Chem. Phys. 89, 947 (1988).

${ }^{64}$ M. J. Bramley, J. W. Tromp, T. Carrington, and G. C. Corey, J. Chem. Phys. 100, 6175 (1994).

${ }^{65}$ G. Ma, R. Chen, and H. Guo, J. Chem. Phys. 110, 8408 (1999).

${ }^{66}$ E. G. Vergini, J. Phys. A: Math. Gen. 37, 6507 (2004). 